\title{
Experimental evidence for the benefits of higher X-ray energies for macromolecular crystallography
}

S. L. S. Storm ${ }^{1, *}$, D. Axford ${ }^{1} \&$ R. L. Owen ${ }^{1,2}$

${ }^{1}$ Diamond Light Source, Harwell Science and Innovation Campus, Didcot OX11 0DE, United Kingdom

${ }^{2}$ robin.owen@diamond.ac.uk

* current address: European Molecular Biology Laboratory, Hamburg Outstation c/o DESY, Notkestraße 85, 22603 Hamburg, Germany 


\begin{abstract}
$\mathrm{X}$-ray induced radiation damage is a limiting factor for the macromolecular crystallographer and data must often be merged from many crystals to yield complete datasets for structure solution of challenging samples. Increasing the X-ray energy beyond the typical 10-15 keV range promises to provide an extension of crystal lifetime via an increase in diffraction efficiency. To date however hardware limitations have negated any possible gains. Through the first use of a Cadmium Telluride Eiger2 detector and a beamline optimised for high energy data collection, we show that at higher energies fewer crystals will be required to obtain complete data, as the diffracted intensity per unit dose increases by a factor of more than 3 between 12.4 and $25 \mathrm{keV}$. Additionally, those higher energy data provide more information, evidenced by an increase in high-resolution limit of up to $0.3 \AA$, pointing to a high energy future for synchrotron-based macromolecular crystallography.
\end{abstract}




\section{Introduction}

Synchrotron-based macromolecular crystallography (MX) is the method of choice for determining the atomic structure of proteins and viruses, providing almost $90 \%$ of Protein Data Bank depositions over the last 5 years ${ }^{1}$. More than $95 \%$ of these synchrotron-derived depositions were collected using X-rays with energies in the range 10-15 keV $(1.240-0.827$ $\AA$ ) reflecting the optimisation of sources, beamlines, and detectors within this narrow region and, on the sample side, the success of seleno-methionine incorporation for experimental phasing at $12.67 \mathrm{keV}^{2}$. The continual development of synchrotron beamlines and sources has resulted in the realization of smaller beam sizes and increased flux densities at the sample position $^{3}$. While these brighter beams enable structure solution from ever-smaller and more challenging crystals it is at the expense of the one-crystal one-structure approach as X-ray induced damage precludes the collection of a complete dataset from a single crystal ${ }^{4}$. In such cases, formation of a complete dataset is achieved using a multi-crystal methodology, distributing the total dose required for structure solution over many crystals ${ }^{5,6}$.

Robust approaches for both collecting and processing multi-crystal data have been developed $^{5,7-11}$ with the logical endpoint being serial synchrotron crystallography where a single diffraction image is collected from each crystal ${ }^{12}$. Rather than collecting from ever more crystals however a primary aim of a multi-crystal experiment should be to optimize the last experimental step, maximising the volume of data that can be collected from each crystal, reducing sample consumption and simplifying data collection and subsequent analysis.

Increasing the energy of the incident X-rays as a solution to the multi-crystal challenge is attractive as no change to, or treatment of, the crystals used is required to achieve the change and the approach is universally applicable as it exploits the differing energy dependence of how X-rays interact with matter via elastic scattering, inelastic scattering or the photoelectric effect. The resulting benefits of higher energies are two-fold. Firstly, as the X-ray energy 
increases the number of elastically scattered photons per unit absorbed dose, or Diffraction Efficiency (DE), increases which is reflected experimentally in higher diffraction intensities for a given dose (improved I/D ratio) $)^{13-15}$ meaning crystal exposures can be reduced. Secondly, at higher energies photoelectron escape means energy deposited by X-rays can leave the crystal, depending on its volume, further reducing the absorbed dose ${ }^{16}$. A key result from many years of work on radiation damage to cryo-cooled crystals is that X-ray induced damage is proportional to the absorbed dose ${ }^{17}$. Both of the effects introduced above predict higher diffracted intensities per unit absorbed dose at higher energies thus the dose can be reduced to obtain the same diffraction intensities. Consequently, the use of higher X-ray energies implies that more useful diffraction data can be collected from each crystal.

The energy dependence of DE was first noted by Arndt (1984), who showed that for crystalline proteins the probability of photoelectric absorption decreases more rapidly with increasing photon energy than the probability of elastic scattering. The intensity of Bragg spots can be predicted using Darwin's equation ${ }^{18}$, which takes X-ray beam and crystal parameters into account. A closer inspection of this also reveals a resolution dependence ${ }^{19}$ with gains in DE at higher X-ray energies enhanced for higher resolution reflections.

In addition to the decrease in photoelectric absorption at high energies, another effect decreasing the deposited dose at higher energies is photoelectron escape. Nave \& Hill (2005) simulated the track of photoelectrons in micro-crystals and concluded that a significant proportion of photoelectrons could leave the crystal before causing damage ${ }^{16}$. The inclusion of photoelectron escape and Compton scattering into calculation of the diffraction efficiency shows a theoretical five-fold gain in DE for $5 \mu \mathrm{m}$ crystals when the energy of incident X-rays is increased from 7 to $30 \mathrm{keV}^{20}$.

To date attempts to demonstrate the benefits of high energy data collection on protein crystals have been hampered by an absence of suitable detectors. Most experiments performed 
at high energies have been performed with CCD detectors ${ }^{14,21,22}$ partially combined with X-ray image intensifiers ${ }^{23}$, image plates ${ }^{21,24-26}$ or even with point detectors ${ }^{27}$. All studies performed with two-dimensional detectors report that the detective quantum efficiency is rather low and not well characterized for high energies. Currently, the most widely applied detector technology for recording diffraction data at synchrotrons utileses a hybrid photon counting (HPC) approach. HPC detectors feature a sensor bonded to an electronic counter and X-ray photons absorbed by the sensor material are recorded as a 'count' ${ }^{28}$. Typically the sensor material is silicon, a widely available, easily processible material. As the atomic number of silicon is low, the sensor rapidly becomes transparent as the X-ray energy is increased beyond $15 \mathrm{keV}$ however. This decrease in absorption means the quantum efficiency (QE) of siliconbased detectors falls rapidly as a function of energy: to less than $20 \%$ at $25 \mathrm{keV}$ for a $450 \mu \mathrm{m}$ thick Si sensor ${ }^{29}$. Recently, detectors using cadmium telluride as a sensor material have been developed; the use of CdTe results in a detector QE of more than $90 \%$ below the cadmium absorption edge $(26.7 \mathrm{keV})$ and nearly $80 \%$ up to energies of $80 \mathrm{keV}^{30}$. Simulations with RADDOSE-3D taking into account the quantum efficiency for a detector with a $750 \mu \mathrm{m}$ thick CdTe sensor and assuming a top-hat beam profile predict an optimal data collection energy of $26 \mathrm{keV}^{31}$.Initial experiments with a Pilatus3 detector equipped with a CdTe sensor were recently successfully used in MX to experimentally prove the benefits of photoelectron escape in micro-crystals ${ }^{32}$, to collect data at $35 \mathrm{keV}$ to ultra-high resolution ${ }^{33}$ and to investigate specific radiation damage at high energies ${ }^{34}$.

Here we present the first use of a CdTe Eiger2 detector for routine high energy MX. The detector provides a nearly constant quantum efficiency across the investigated energy range. We experimentally demonstrate increased dose efficiency at higher energies observing an almost four-fold increase between 12.4 and $25 \mathrm{keV}$. No gain is observed when an identical experimental approach is employed using a silicon detector. We further observe an increase in 
the resolution of data obtained for a given absorbed dose at higher energies. The combination of these effects will allow fewer crystals to be used for structure determination and our results point to a high energy future for synchrotron-based MX.

\section{Results and Discussion}

In order to quantify the energy dependence of the diffraction efficiency of protein crystals, 29 low-dose data series were collected from 11 thermolysin crystals. Each data series consisted of four $100^{\circ}$ wedges collected from the same position on a crystal with each wedge recorded using a different X-ray energy (12.4 keV, $17.5 \mathrm{keV}, 22.3 \mathrm{keV}$ or $25 \mathrm{keV}) .22$ data series were recorded using an Eiger2 9M (750 $\mu \mathrm{m}$ thick CdTe sensor) and 7 with a Pilatus3 6M (450 $\mu \mathrm{m}$ thick Si sensor) detector. In order to minimize the contribution of radiation damage to observed trends, the total absorbed dose was kept low: to less than 800 Gy per sweep i.e. less than $2 \%$ of the 43 MGy required to half the diffracting power of cryo-cooled crystals ${ }^{35}$ and the order of energies varied between each series (table S1).

Figure 1 shows the agreement between the observed diffracted intensity divided by the beam intensity $\left(\mathrm{I} / \mathrm{I}_{\text {beam }}\right)$ as a function of energy for the crystals used in this study and $\mathrm{I} / \mathrm{I}_{\text {beam }}$ predicted by Darwin's equation which is presented in a simple form by Giocovazzo ${ }^{36}$

$$
\frac{I}{I_{\text {beam }}}=k_{1} k_{2} L P T E\left|F_{H}\right|^{2}
$$

Here $k_{1}=e^{4} / m^{2} c^{4}$, takes into account universal constants, and $k_{2}=\lambda^{3} V_{x t a l} / V_{\text {cell }}^{2}$ where $\lambda$ is the X-ray wavelength, $V_{\text {xtal }}$ the illuminated crystal volume and $V_{\text {cell }}$ the volume of the unit cell. $L$ is the Lorentz factor, $P$ the polarization factor, and $T$ and $E$ the X-ray transmission and extinction coefficients of the crystal respectively. The analytical expression for the product of $<\mathrm{LP}>\mathrm{f}_{\mathrm{obs}}=\pi \frac{(3+\cos 4 \theta)}{16 \sin \theta}$ derived by Holton ${ }^{20}$ can be used, and energy independent terms excluded to show 


$$
\frac{I}{I_{\text {beam }}} \propto V_{x t a l} \lambda^{3} \frac{(3+\cos 4 \theta)}{\sin \theta} e^{-\mu_{a b s} t}
$$

where $\theta$ is the Bragg angle, $t$ the crystal thickness and $\mu_{a b s}$ its X-ray absorption coefficient. When applying this expression to the data collected here we also consider the energy dependent quantum efficiency of the detector used.

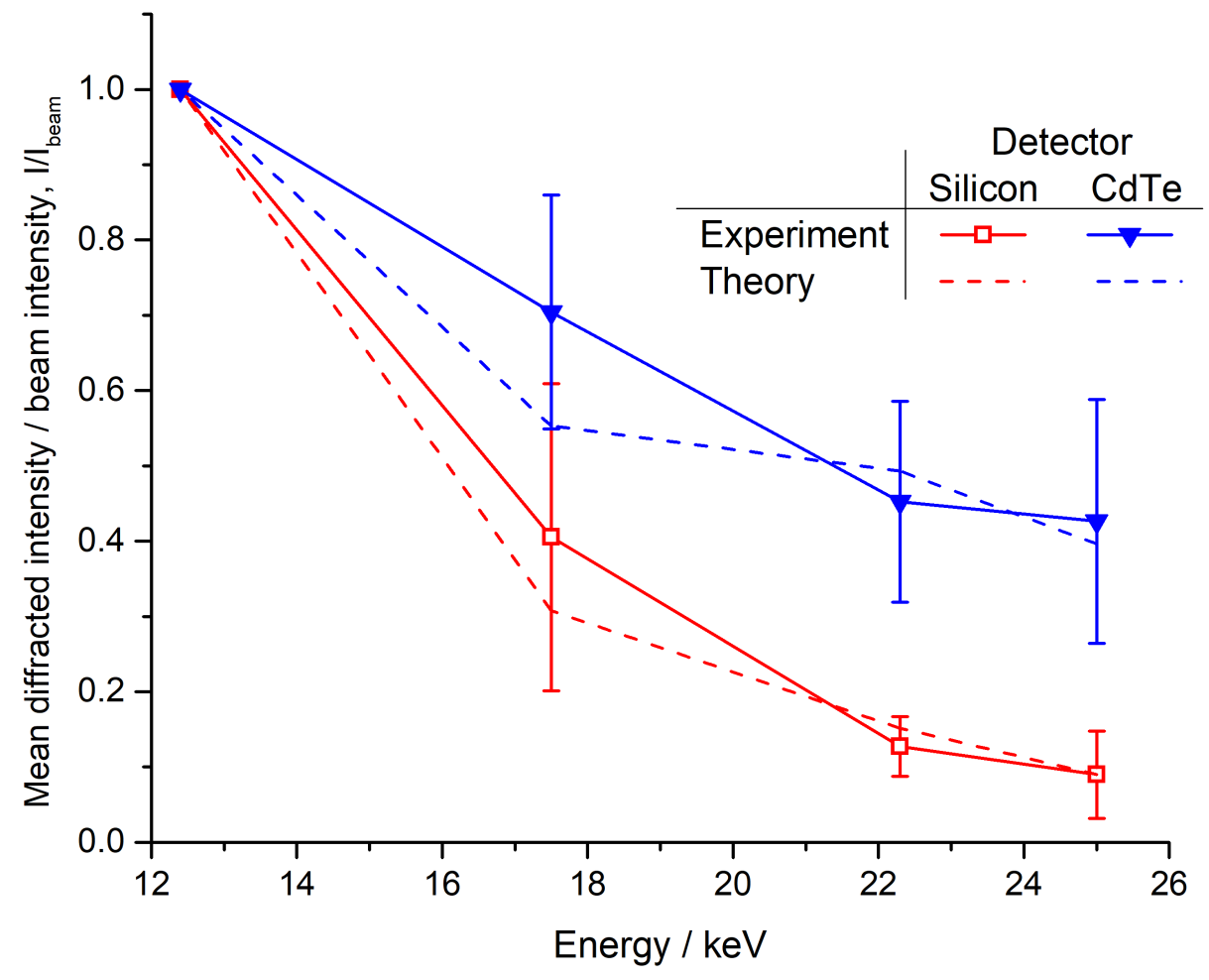

Figure 1. Ratio of diffracted intensity to beam intensity (I/I $\left.I_{\text {beam }}\right)$ as a function of energy for data recorded using both the CdTe Eiger and Si Pilatus detectors. Experimental data are the mean of 22 and 7 data sets for the Eiger and Pilatus detectors respectively. Overlaid is the predicted energy dependence of I/I beam determined from theory using measured fluxes and beamsizes. 
The agreement between experiment and theory shown in figure 1 illustrates that the ratio of elastically scattered photons to incident photons varies as expected for both detectors and, importantly, gives confidence in both the accuracy and validity of the quantity mean diffracted intensity as the numerator I in the ratio I/D derived below.

Intensity statistics for two individual data series collected from the same crystal, one recorded using each detector, are shown in figure 2. Both of these data series were collected with the same data collection parameters and the same increasing energy sequence $(12.4 \mathrm{keV}$, $17.5 \mathrm{keV} 22.3 \mathrm{keV}$ and finally $25 \mathrm{keV}$ ). The mean unscaled intensities recorded over the two data series while aiming at keeping the total diffracted intensity approximately constant are shown in figure 2a. A strong energy dependence is clear for data recorded using the CdTe Eiger detector with higher intensities recorded at higher energies, a trend not observed using the Si Pilatus. The observed signal to noise ratio, $<\mathrm{I} / \sigma(\mathrm{I})>$, is shown in figure $2 \mathrm{~b}$, this illustrates again the advantage conferred by cadmium telluride. CdTe data again show a constant increase as a function of energy whereas the maxima for Si data is at $17.5 \mathrm{keV}$ : at 22.3 and $25 \mathrm{keV}$ when the absorption of silicon is low $<\mathrm{I} / \sigma(\mathrm{I})>$ falls. Concomitant with the decrease in the quantum efficiency of the silicon sensor, the internal consistency of the data also decreases, as can be seen from the increased $\mathrm{R}_{\text {meas }}$ values for data collected with the Si Pilatus detector at higher energies (figure 2c). We note that other detector properties beyond the sensor material contribute to some of the observed differences: both the smaller pixel size and zero deadtime of the Eiger detector also affect data quality ${ }^{37}$. The $0.95 \mathrm{~ms}$ dead time of the Pilatus detector used corresponds to almost $10 \%$ of the total exposure time for data collected at energies below $25 \mathrm{keV}$ for example. Our results clearly show however that the CdTe sensor material is essential to exploit the benefits of high energy data collection.

With doses of less than $540 \mathrm{kGy}$ per data set (table S2) global radiation damage is unlikely to have a significant effect on the observed trends. Indeed 2Fo-Fc maps comparing 
bioRxiv preprint doi: https://doi.org/10.1101/2021.01.21.427633; this version posted January 21,2021 . The copyright holder for this preprint (which was not certified by peer review) is the author/funder, who has granted bioRxiv a license to display the preprint in perpetuity. It is made available under aCC-BY-NC-ND 4.0 International license.

data sets collected from the same position in the crystal display almost no signs of site-specific radiation damage (figure $\mathrm{S} 1$ ).

(a)

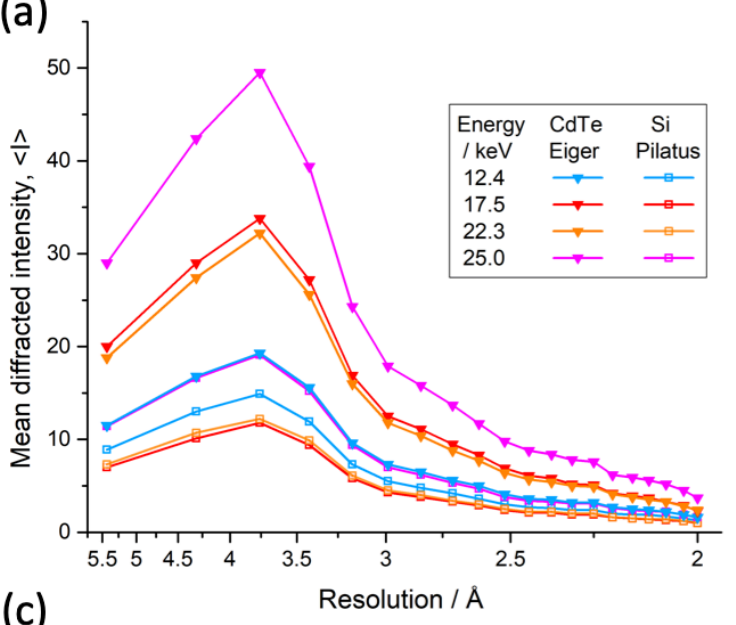

(c)

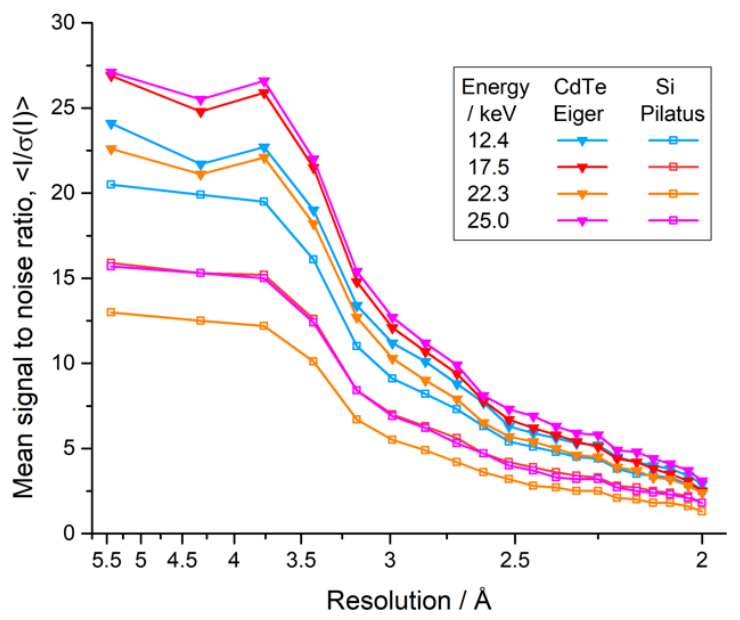

(b)

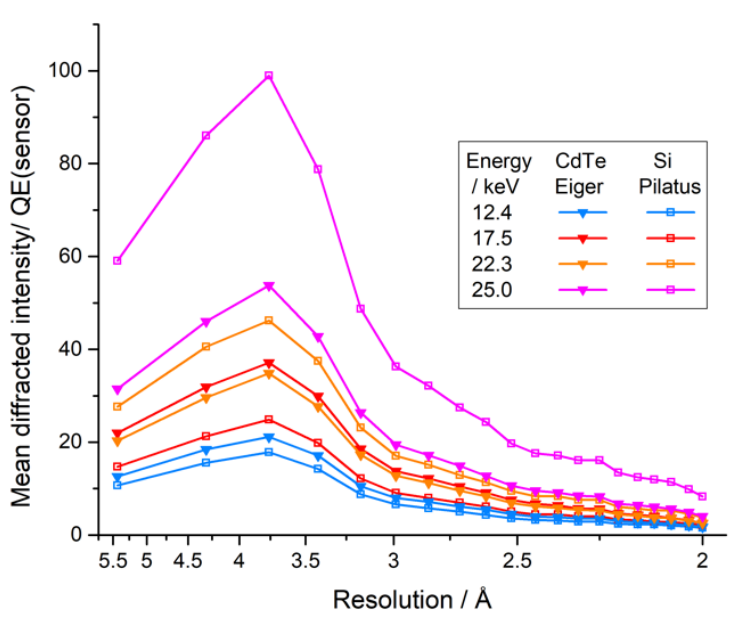

(d)

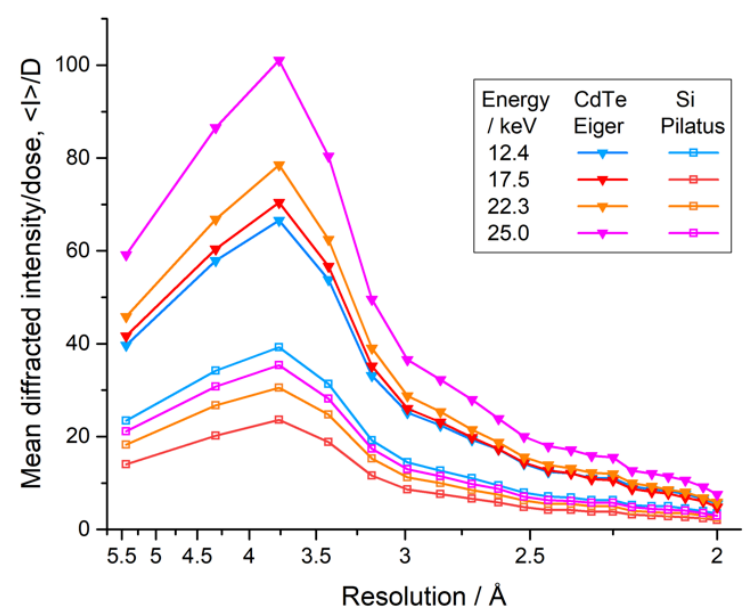

Figure 2: Overview of two data series collected from the same crystal using a CdTe Eiger detector (filled triangles) and a Si Pilatus detector (hollow squares). (a) shows the mean unscaled intensity per Bragg spot $<I>$, as a function of resolution (b) Mean unscaled signal to noise ratio per Bragg spot $<I / \sigma(I)>$. (c) Redundancy independent merging $R$ value $R_{\text {meas. }}$ (d) Mean diffracted intensity per Bragg spot normalized to the absorbed dose, $<I>/ D$. In all panels data points reflect the high resolution limit of each shell, the lowest resolution data point includes reflections over the range $40-5.5$ A. 
Figure $2 \mathrm{~d}$ shows the diffracted intensity per unit absorbed dose, $\langle\mathrm{I}>/ \mathrm{D}$, for the two data series. When the CdTe Eiger detector, optimised for high energy data collection, is used a clear increase in $<\mathrm{I}>/ \mathrm{D}$ is observed at 22.3 and $25 \mathrm{keV}$. The trend of increasing diffracted intensity per unit absorbed dose as a function of energy was observed for all crystals: over 22 data series the mean increase in $<\mathrm{I}>/ \mathrm{D}$ between 12.4 and $25 \mathrm{keV}$ is of 3.35 (figure 3). When using a detector with a silicon sensor, no such increase is observed with $<\mathrm{I}>/ \mathrm{D}$ approximately constant over the energy range used. Five additional data series were collected from two crystals over a higher X-ray energy range $(22.3-27 \mathrm{keV})$ as the optimal energy for data collection using a CdTe based detector is expected to be at around $26 \mathrm{keV}^{31}$ Over this limited energy range we observe no clear peak in the diffracted intensity per unit dose with $<\mathrm{I}>/ \mathrm{D}$ approximately constant between 22 and $26 \mathrm{keV}$, though a significant decrease, beyond experimental error, is observed at $27 \mathrm{keV}$ as expected (figure 3 inset). 


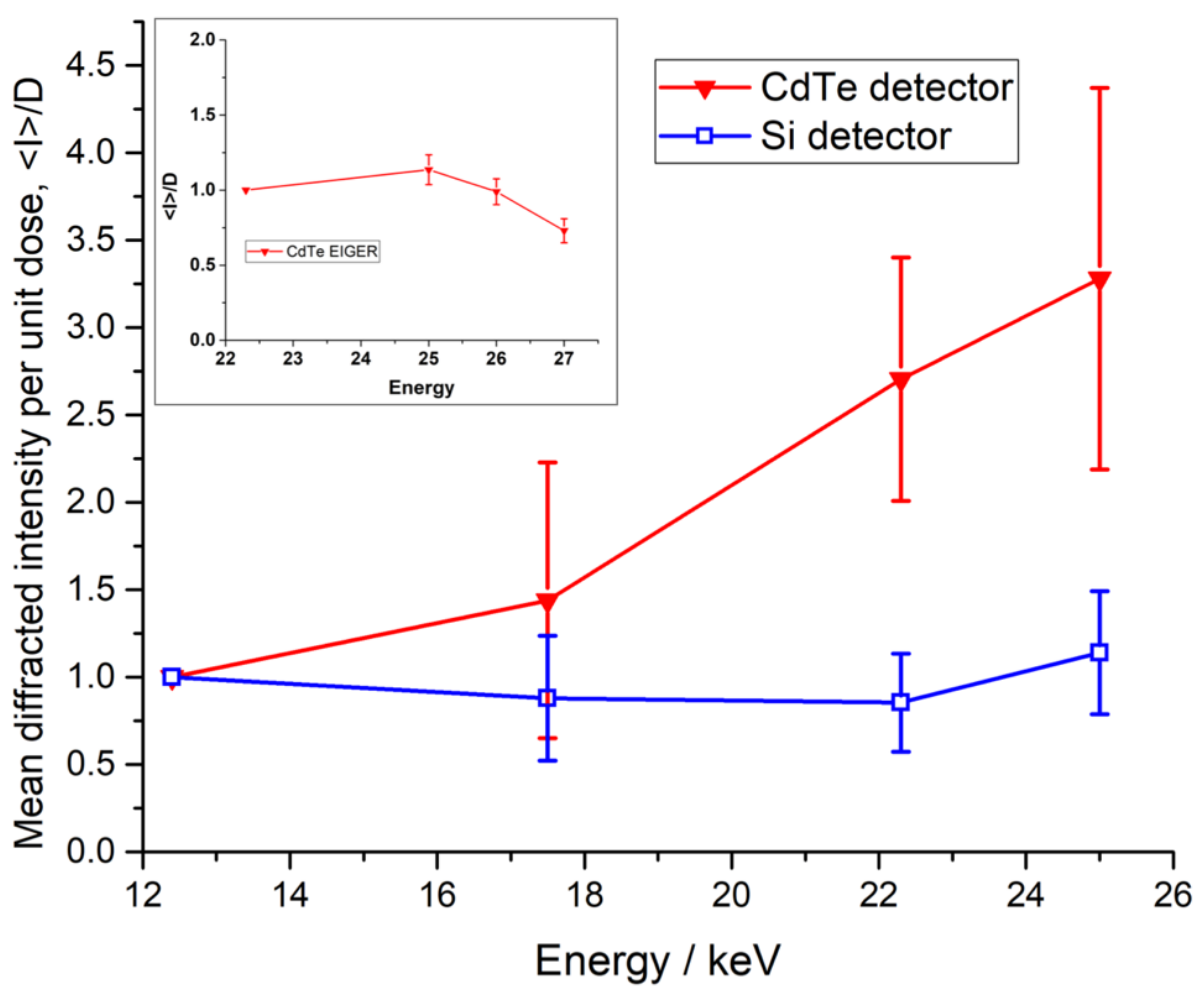

Figure 3: Increase in the diffracted intensity per unit absorbed dose, $\langle I\rangle / D$, as a function of energy. Data shown are averaged from 29 data series (22 recorded using CdTe Eiger and 7 using the Si Pilatus) with the standard deviation at each energy shown as error bars. Variation in $<I>/ D$ over an energy range of 22.3-27 keV observed in additional 5 data series is shown in the inset. These data are normalised to $22.3 \mathrm{keV}$.

While an increase in diffraction efficiency as a function of energy is predicted by theory ${ }^{13}$, the size of the gain observed in these experiments is larger than predicted ${ }^{31}$ by a factor of $\sim 2$. There are other experimental factors which act to increase the gains realized for high energy data collection. Firstly, the beam used in these experiments has a Gaussian rather than top-hat profile. The theoretical increase of 1.6 in DE between 12.4 and $25 \mathrm{keV}$ predicted by Dickerson and Garman ${ }^{31}$ assumes a top-hat beam, with gains of 3 to 4 only realized for beam and crystal sizes of less than 2 microns. When a Gaussian beam is used, photoelectrons will not be generated evenly within the illuminated volume, which is partly reflected in an increased theoretical diffraction efficiency of 1.9 between 12.4 and $25 \mathrm{keV}$ when this is factored into the 
dose calculation. Some additional gain may therefore result as photoelectrons migrate out of the central high dose region, aided by the longer path lengths of photoelectrons at higher energies. Secondly, one has to consider that, despite best efforts, small errors in the measurement of the X-ray beam size and intensity and crystal size can easily result in under or over-estimation of the absorbed dose and hence I/D. We sought to minimise this as a source of error through the use of multiple crystals and data collection over multiple sessions with the flux and beamsize measured at each.

Some studies postulate that intensities at higher energies can be enhanced relative to lower energies due to a reduced background and lower absorption ${ }^{38,39}$. Here, we choose to keep the resolution at detector edge constant, moving the detector further away from the crystal at higher energies, since this is how crystallographers will best exploit the energy dependent gains for real-world data collection. This increase in crystal to detector distance has an influence on the data measured and an increase in both mean spot size and background intensity is observed with increasing energy. Spot size is heavily dependent on beam divergence, hence a longer sample-to-detector distance at higher incident beam energy will lead to a larger spot on the detector surface. With the CdTe Eiger detector an increase in measured background was seen at higher energies, consistent with elastic scattering from the non-crystalline component of the sample being the main factor as it is concentrated at smaller angles with increasing beam energy $^{26}$. Our observations suggest that variation in spot size and background intensity are not significant influences on improvements in the metrics of diffraction data quality seen at higher energies.

In the above, photoelectron escape has not been considered when calculating absorbed doses. While this effect is negligible at $12.4 \mathrm{keV}$, at higher energies photoelectrons can escape from the illuminated volume reducing the effective deposited dose. At $25 \mathrm{keV}$ the desposited dose is reduced by $20 \%$ for $20 \mu \mathrm{m}$ crystals to $\mathrm{D}_{\mathrm{PE}}\left(\mathrm{D}\right.$ and $\mathrm{D}_{\mathrm{PE}}$ calculated using $R A D D O S E-3 D$ 
and shown in table S2). Compared with three-fold increase in $<\mathrm{I}>/ \mathrm{D}$ shown in figure $3,<\mathrm{I}>/ \mathrm{DPE}$ shows a further increase as a function of energy, increasing by a factor of 4 between 12.4 and $25 \mathrm{keV}$ (figure S4). Initial experiments with microcrystals of a volume smaller than the smallest available beamsize of $7 \mu \mathrm{m} \times 8 \mu \mathrm{m}$ show that these effects amount can amount for a factor of 6 over this energy range (data not shown).

To experimentally confirm the predicted resolution dependence of the gains in diffracted intensity per unit dose, high resolution (1.25 $\AA$ ) data series were also collected. The dimensions of the 9M Eiger and the currently achievable minimum crystal to detector distance at I24 preclude data collection to this resolution at $12.4 \mathrm{keV}$ so these data series were normalized to $17.5 \mathrm{keV}$. Figure 4a shows the mean diffracting power per unit absorbed dose, $<\mathrm{I}>/ \mathrm{D}$, in different resolution shells as a function of energy for data recorded using the CdTe Eiger. A clear resolution dependence is observed with $<\mathrm{I}>/ \mathrm{D}$ increasing by a factor of 2.7 for the lowest resolution shell compared to 4.2-fold over the range 1.35-1.25 $\AA$. It follows that the high resolution limit of the diffraction data should also increase at higher energies. Figure $4 \mathrm{~b}$ shows the energy dependent resolution cut-off for five crystals diffracting to between 2.1 and $1.65 \AA$ at $12.4 \mathrm{keV}$. The resolution limit was determined using dials.scale applying a cut-off criterion of $\mathrm{CC}_{1 / 2}>0.3$. For all crystals, an energy dependent increase in the resolution cut-off is observed with a gain of between 0.2 and $0.3 \AA$. The resolution gain is also clearly visible in the electron density maps (figure S2). Reduced background and lower absorption at high Xray energies also act to explain the gain in resolution. 
(a)
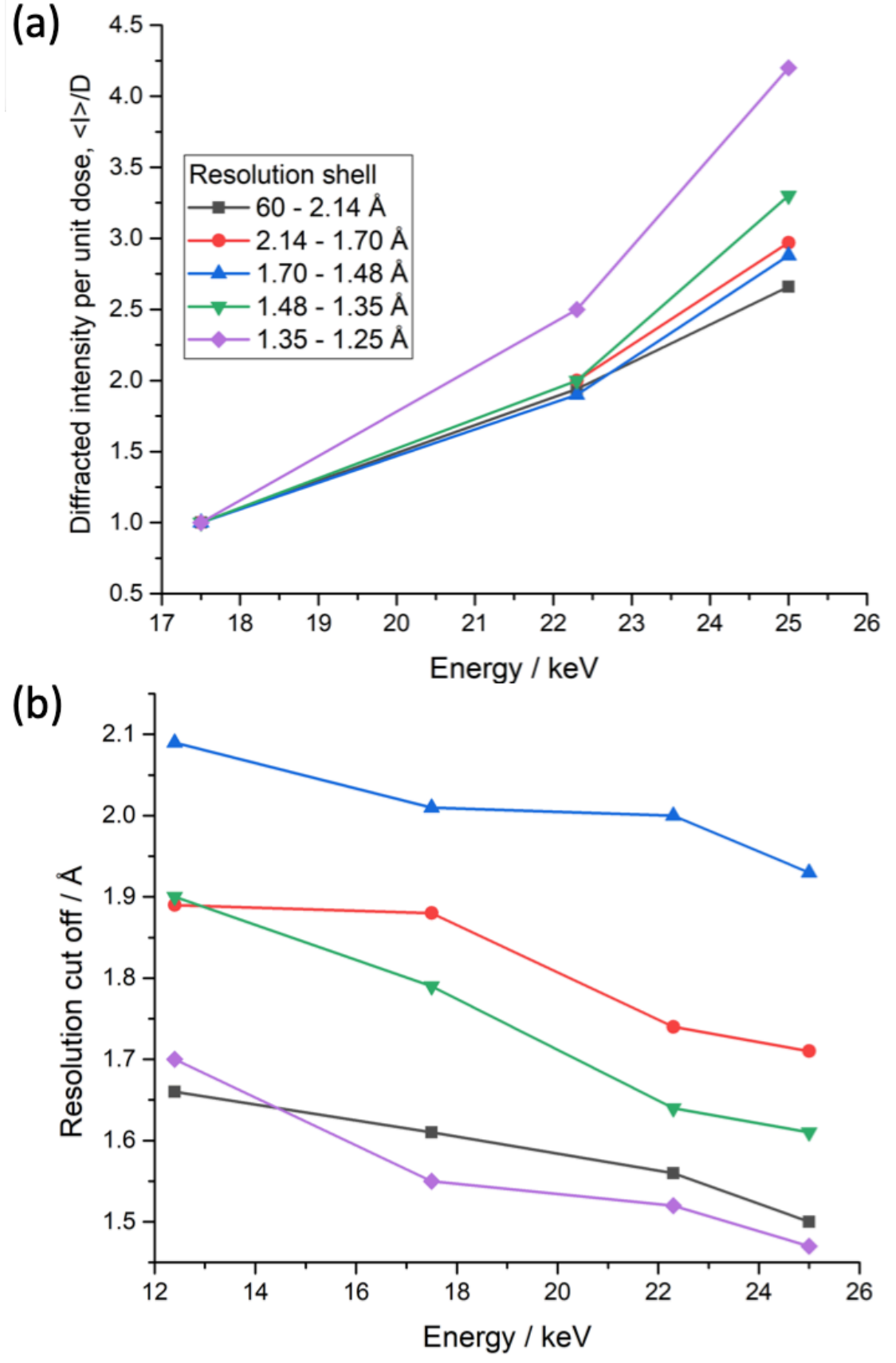

Figure 4: (a) Resolution dependence of the diffracted intensity per unit absorbed dose, $<I>/ D$, and (b) energy dependent change in resolution cutoff as ascertained using a criterion of $C C_{1 / 2}$ $>0.3$.

With the advancement of synchrotron technology, from undulators providing high photon fluxes at high energies through to large area detectors able to efficiently record highenergy photons, it is now possible to efficiently and routinely collect data at energies which are optimal for macromolecular crystallography. Within this study, we show experimentally 
that a CdTe based detector enables the increased diffraction efficiency of crystals at high Xray energies to be exploited due to its increased quantum efficiency in comparison to siliconbased detectors. The energy dependence of intensities follows Darwin's law in both cases. The benefits of collecting at $25 \mathrm{keV}$ include an increase in the resolution of data up to $0.3 \AA$ that can be recorded for a given absorbed dose, compared to data collected at $12.4 \mathrm{keV}$. This increase in information content can for example enable the identification of water molecules in structural enzymology and make a critical difference in understanding structural function of proteins.

These results impact all macromolecular crystallography experiments from rotation to serial even when the crystal sizes used are relatively modest ( $\sim 20$ microns) and should be considered in the design of future MX beamlines and for X-ray data collection from all samples that yield crystals of limited size.

\section{Methods}

\section{Sample preparation and sample mounting}

Thermolysin crystals were grown in CrystalQuickX 96 well sitting drop plates (Greiner) using a Mosquito crystallization robot (STP Labtech) at $20^{\circ} \mathrm{C} .50 \mathrm{mg} / \mathrm{ml}$ lyophilized thermolysin (Sigma) was dissolved in $0.05 \mathrm{M}$ MES pH 6, $45 \%$ DMSO, $50 \mathrm{mM} \mathrm{NaCl}$ and equilibrated against 1.2 $\mathrm{M}$ ammonium sulphate in a 1:1 ratio with a final drop size of $200 \mathrm{nl} .50 \%$ Ethylene glycol or $3 \mathrm{M}$ ammonium sulphate were used as cryo-protectant. Crystals were mounted in loops and then cryo-cooled. Dimensions of the rod-shaped crystals were measured using the data acquisition GUI (GDA) and ranged from $20-40 \mu \mathrm{m}$ in diameter with lengths of 120-310 $\mu \mathrm{m}$. 


\section{Beamline setup}

All experiments were carried out at beamline I24 at Diamond Light Source. A new cryogenic permanent magnet undulator (CPMU) was installed shortly before the first experiments described here. Commissioning resulted in a varying X-ray flux between each experimental session. The X-ray flux was measured using a PD300-500 silicon PIN diode ${ }^{40}$ (Canberra), built by the Diamond Light source detector group and calibrated by the Physikalisch-Technische Bundesanstalt (PTB) up to energies of $60 \mathrm{keV}$, at the start of each session and is given in table S1. I24 features a two-stage focusing design with two pairs of Kirkpatrick-Baez mirrors, the first pair of mirrors features stripes with different coatings. For data collections below $20 \mathrm{keV}$ a rhodium stripe was used, for experiments above $20 \mathrm{keV}$ a platinum stripe were used. As the shape of mirrors was optimised at $12.4 \mathrm{keV}$, the beamsize increased when the Pt strip was used. The secondary pair of mirrors were not translated during the experiments as the $\mathrm{Rh}$ and $\mathrm{Pt}$ stripes overlap. Beam sizes at the sample position were determined by performing a knife-edge scan on a $200 \mu \mathrm{m}$ thick gold wire and FWHM are given in table S1. Care was taken to measure beamline variables such as beamsize and the photon flux prior to each data collection session.

\section{Detector setup}

Data were collected using an Eiger $2 \mathrm{X} 9 \mathrm{M}$ detector with a $750 \mu \mathrm{m}$ thick CdTe sensor and a Pilatus $3 \mathrm{X} 6 \mathrm{M}$ with a $450 \mu \mathrm{m}$ thick silicon sensor. The maximum frame rate of the Pilatus detector is $100 \mathrm{~Hz}$ (10 ms exposure times), while the Eiger detector can run at $230 \mathrm{~Hz}(4.3 \mathrm{~ms})$. The detectors are mounted in an up and under configuration on a translation stage allowing data to be collected from each interchangeably. The sensor quantum efficiencies of each detector were interpolated from measured values provided by Dectris (Baden, Switzerland).

\section{Data collection}

For each data series diffraction data were collected at 12.4, 17.5, 22.3 and $25 \mathrm{keV}$ at a single position on a crystal. The order in which each energy was collected was varied between each 
data series. Doses were low and ranged between 300 and $800 \mathrm{kGy}$ per dataset. At each position on a crystal repeated wedges of $100^{\circ}$ of diffraction data were collected, with a single wedge at each energy. The crystal to detector distance was varied such that the inscribed circle on the detector face corresponded to the same resolution at all energies. When multiple data series were collected from a single crystal the crystal was translated by at least twice the beam FWHM between each series.

To enable direct comparison of the performance of the CdTe Eiger detector to that of the Si Pilatus detector, the exposure time was set to $10 \mathrm{~ms}$ in session A for the data collected at $22.3 \mathrm{keV}$ or below. In subsequent sessions when the Pilatus was not used for comparison, an exposure time of $5 \mathrm{~ms}$ was used. In session A, at least one set of collections at the four energies was performed with both detectors from the same crystal. For the data at $25 \mathrm{keV}$, exposure times up to $83 \mathrm{~ms}$ at full transmission were required to compensate for the lower flux as well as the lower scattering efficiency. To be able to calculate the flux as accurately as possible, intensity values from a X-ray beam position monitor close to the sample position were recorded for all datasets. In session $\mathrm{D}$, higher energies became accessible due to the ongoing commissioning of the CPMU, though exposures of $0.7 \mathrm{~s}$ per data frame were required at $27 \mathrm{keV}$. As commissioning continues it is anticipated that higher fluxes, and hence reduced exposure times, will become accessible with any time penalty associated with high energy data collection removed. To allow the energy dependence of $<\mathrm{I}>/ \mathrm{D}$ at high resolution to be probed, exposure times were increased and a shorter crystal to detector distance used during this session.

\section{Data processing}

Data were processed with DIALS v.3.0.4 integration package ${ }^{41}$. To generate statistics with consistent parameters across data sets, $x i a 2^{42}$ was used. Raw intensities were used from the 
data processing integration step to avoid complications introduced by scaling routines or inadvertent 'scaling out' of energy dependent differences.

Crystals typically diffracted to $1.4-2.0 \AA$, taking into account diffraction in the corner of the detector and applying the $\mathrm{CC}_{1 / 2}>0.33$ criterion. However, a resolution cut-off of $2.0 \AA$ was applied to all data when quantifying energy dependent changes in the mean diffracting power. This was defined by the maximum resolution of the inscribed circle at the minimum detector distance at $12.4 \mathrm{keV}$.

The average diffraction weighted dose, referred to as dose here, was calculated for each dataset using $R A D D O S E-3 D^{43}$. Dose calculations assumed that the long axis of the crystal is oriented vertically towards the X-ray beam. The $<\mathrm{I}>/ \mathrm{D}$ value is defined as to the unscaled mean intensity given by xia2 from the lowest resolution shell up to $2.0 \AA$ divided by the deposited dose estimated for the individual data set.

\section{Acknowledgements}

We would like to thank Halina Mikolajek and Sam Horrell for producing the thermolysin crystals. Andrew Foster, James O’Hea, Scott Williams and Adam Taylor are thanked for setting up the CdTe Eiger detector on I24. Furthermore, we thank Colin Nave for his very helpful comments on the manuscript and James Holton for enlightening discussions on Darwin's equation.

\section{Author contributions}

S.L.S.S. planned the experiment with contributions from R.L.O. and D.A. The data collection was performed by all authors. Most of the data analysis was done by S.L.S.S. with support from R.L.O and D.A.. The manuscript was written by S.L.S.S., D.A. and R.L.O. 


\section{References}

1. Goodsell, D. S. et al. RCSB Protein Data Bank: Enabling biomedical research and drug discovery. Protein Sci. 29, 52-65 (2020).

2. Hendrickson, W. A., Horton, J. R. \& LeMaster, D. M. Selenomethionyl proteins produced for analysis by multiwavelength anomalous diffraction (MAD): a vehicle for direct determination of three-dimensional structure. EMBO J. 9, 1665-1672 (1990).

3. Owen, R. L., Juanhuix, J. \& Fuchs, M. Current advances in synchrotron radiation instrumentation for MX experiments. Arch. Biochem. Biophys. 602, 21-31 (2016).

4. Smith, J. L., Fischetti, R. F. \& Yamamoto, M. Micro-crystallography comes of age. Curr. Opin. Struct. Biol. 22, 602-612 (2012).

5. Liu, Q., Zhang, Z. \& Hendrickson, W. A. Multi-crystal anomalous diffraction for low-resolution macromolecular phasing. Acta Crystallogr. D Biol. Crystallogr. 67, 45-59 (2011).

6. Yamamoto, M. et al. Protein microcrystallography using synchrotron radiation. IUCrJ 4, 529-539 (2017).

7. Giordano, R., Leal, R. M. F., Bourenkov, G. P., McSweeney, S. \& Popov, A. N. The application of hierarchical cluster analysis to the selection of isomorphous crystals. Acta Crystallogr. D Biol. Crystallogr. 68, 649-658 (2012).

8. Foadi, J. et al. Clustering procedures for the optimal selection of data sets from multiple crystals in macromolecular crystallography. Acta Crystallogr. D Biol. Crystallogr. 69, 1617-1632 (2013).

9. Zander, U. et al. MeshAndCollect : an automated multi-crystal data-collection workflow for synchrotron macromolecular crystallography beamlines. Acta Crystallogr. D Biol. Crystallogr. 71, $2328-2343$ (2015).

10. Santoni, G., Zander, U., Mueller-Dieckmann, C., Leonard, G. \& Popov, A. Hierarchical clustering for multiple-crystal macromolecular crystallography experiments: the ccCluster program. J. Appl. Crystallogr. 50, 1844-1851 (2017). 
11. Yamashita, K., Hirata, K. \& Yamamoto, M. KAMO : towards automated data processing for microcrystals. Acta Crystallogr. Sect. Struct. Biol. 74, 441-449 (2018).

12. Diederichs, K. \& Wang, M. Serial Synchrotron X-Ray Crystallography (SSX). Protein Crystallogr. 239-272 (2017) doi:10.1007/978-1-4939-7000-1_10.

13. Arndt, U. W. Optimum X-ray wavelength for protein crystallography. J. Appl. Crystallogr. 17, 118-119 (1984).

14. Fourme, R. et al. Reduction of radiation damage and other benefits of short wavelengths for macromolecular crystallography data collection. J. Appl. Crystallogr. 45, 652-661 (2012).

15. Helliwell, J. R., Ealick, S., Doing, P., Irving, T. \& Szebenyi, M. Towards the measurement of ideal data for macromolecular crystallography using synchrotron sources. Acta Crystallogr. D Biol. Crystallogr. 49, 120-128 (1993).

16. Nave, C. \& Hill, M. A. Will reduced radiation damage occur with very small crystals? J. Synchrotron Radiat. 12, 299-303 (2005).

17. Holton, J. M. A beginner's guide to radiation damage. J. Synchrotron Radiat. 16, 133-142 (2009).

18. Darwin, C. G. The reflexion of X-rays from imperfect crystals. Lond. Edinb Dubl Philos Mag J Sci 27, 315-333 (1914).

19. Holton, J. M. \& Frankel, K. A. The minimum crystal size needed for a complete diffraction data set. Acta Crystallogr. D Biol. Crystallogr. 66, 393-408 (2010).

20. Cowan, J. A. \& Nave, C. The optimum conditions to collect X-ray data from very small samples. J. Synchrotron Radiat. 15, 458-462 (2008).

21. Jakoncic, J. et al. Anomalous diffraction at ultra-high energy for protein crystallography. J. Appl. Crystallogr. 39, 831-841 (2006).

22. Shimizu, N., Hirata, K., Hasegawa, K., Ueno, G. \& Yamamoto, M. Dose dependence of radiation damage for protein crystals studied at various X-ray energies. J. Synchrotron Radiat. 14, 4-10 (2007). 
23. Schiltz, M. et al. Protein Crystallography at Ultra-Short Wavelengths: Feasibility Study of Anomalous-Dispersion Experiments at the Xenon K-edge. J. Synchrotron Radiat. 4, 287-297 (1997).

24. Fourme, R. et al. Using a quasi-parallel X-ray beam of ultrashort wavelength for highpressure virus crystallography: implications for standard macromolecular crystallography. Acta Crystallogr. D Biol. Crystallogr. 59, 1767-1772 (2003).

25. Fourme, R. et al. A new paradigm for macromolecular crystallography beamlines derived from high-pressure methodology and results. J. Synchrotron Radiat. 18, 31-36 (2011).

26. Gonzalez, A., Denny, R. \& Nave, C. Data collection at short wavelengths in protein crystallography. Acta Crystallogr. D Biol. Crystallogr. 50, 276-282 (1994).

27. Müller, R., Weckert, E., Zellner, J. \& Drakopoulos, M. Investigation of radiation-dose-induced changes in organic light-atom crystals by accurate $d$-spacing measurements. J. Synchrotron Radiat. 9, 368-374 (2002).

28. Förster, A., Brandstetter, S. \& Schulze-Briese, C. Transforming X-ray detection with hybrid photon counting detectors. Philos. Trans. R. Soc. Math. Phys. Eng. Sci. 377, 20180241 (2019).

29. Donath, T. et al. Characterization of the PILATUS photon-counting pixel detector for X-ray energies from $1.75 \mathrm{keV}$ to $60 \mathrm{keV}$. J. Phys. Conf. Ser. 425, 062001 (2013).

30. Zambon, P. et al. Spectral response characterization of CdTe sensors of different pixel size with the IBEX ASIC. Nucl. Instrum. Methods Phys. Res. Sect. Accel. Spectrometers Detect. Assoc. Equip. 892, 106-113 (2018).

31. Dickerson, J. L. \& Garman, E. F. The potential benefits of using higher X-ray energies for macromolecular crystallography. J Synchrotron Rad 201926 Doi101107S160057751900612X 1-9 (2019) doi:10.1107/S160057751900612X.

32. Storm, S. L. S. et al. Measuring energy-dependent photoelectron escape in microcrystals. IUCrJ 2020 M7 129-135 Doi101107S2052252519016178 1-7 (2020) doi:10.1107/S2052252519016178. 
33. Takaba, K. et al. Subatomic resolution X-ray structures of green fluorescent protein. IUCrJ 6, 387-400 (2019).

34. Ueno, G. et al. Low-dose X-ray structure analysis of cytochrome $c$ oxidase utilizing highenergy X-rays. J. Synchrotron Radiat. 26, 912-921 (2019).

35. Owen, R. L., Rudiño-Piñera, E. \& Garman, E. F. Experimental determination of the radiation dose limit for cryocooled protein crystals. Proc. Natl. Acad. Sci. 103, 4912-4917 (2006).

36. Giacovazzo, C. et al. Fundamentals of Crystallography. 6.

37. Casanas, A. et al. EIGER detector: application in macromolecular crystallography. Acta Crystallogr. Sect. Struct. Biol. 72, 1036-1048 (2016).

38. Fourme, R. et al. Reduction of radiation damage and other benefits of short wavelengths for macromolecular crystallography data collection. J Appl Cryst 201245 652-661 Doi101107S0021889812019164 1-10 (2012) doi:10.1107/S0021889812019164.

39. Helliwell, J. R., Ealick, S., Doing, P., Irving, T. \& Szebenyi, M. Towards the measurement of ideal data for macromolecular crystallography using synchrotron sources. Acta Cryst 1993 D49 120-128 Doi101107S0907444992006747 1-9 (1993) doi:10.1107/S0907444992006747.

40. Owen, R. L., Holton, J. M., Schulze-Briese, C. \& Garman, E. F. Determination of X-ray flux using silicon pin diodes. J. Synchrotron Radiat. 16, 143-151 (2009).

41. Winter, G. et al. DIALS: implementation and evaluation of a new integration package. Acta Cryst 2018 D74 85-97 Doi101107S2059798317017235 1-13 (2018) doi:10.1107/S2059798317017235.

42. Winter, G. xia2 : an expert system for macromolecular crystallography data reduction. J. Appl. Crystallogr. 43, 186-190 (2010).

43. Bury, C. S., Brooks-Bartlett, J. C., Walsh, S. P. \& Garman, E. F. Estimate your dose: RADDOSE3D. Protein Sci. 27, 217-228 (2018). 


\section{Supplementary material}

\begin{tabular}{|c|c|c|c|c|c|c|}
\hline $\begin{array}{l}\text { Session \& } \\
\text { number of } \\
\text { data series }\end{array}$ & $\begin{array}{c}\text { Energy } \\
\text { / keV }\end{array}$ & $\begin{array}{l}\text { Incident flux } \\
/ \times 10^{11} \mathrm{ph} \mathrm{s}^{-1}\end{array}$ & $\begin{array}{c}\text { Beamsize } \\
/ \mu \mathrm{m}^{2}\end{array}$ & $\begin{array}{c}\text { Crystal } \\
\text { diameter } \\
/ \mu \mathrm{m}\end{array}$ & $\begin{array}{c}\text { Total exposure } \\
\text { time per dataset } \\
\text { / s }\end{array}$ & $\begin{array}{c}\text { Total deposited } \\
\text { dose per dataset } \\
\text { / kGy }\end{array}$ \\
\hline \multirow{4}{*}{$\begin{array}{l}\mathbf{A} \\
8 \mathrm{CdTe} \\
7 \mathrm{Si}\end{array}$} & 12.4 & 26.40 & \multirow{2}{*}{$7.9 \times 8.9$} & 25 & 10 & 280 \\
\hline & 17.5 & 8.48 & & 25 & 10 & 440 \\
\hline & 22.3 & 4.09 & \multirow[t]{2}{*}{$9.7 \times 10.2$} & 25 & 10 & 350 \\
\hline & 25.0 & 0.255 & & 25 & 83 & 400 \\
\hline \multirow{4}{*}{$\begin{array}{l}\text { B } \\
8 \mathrm{CdTe}\end{array}$} & 12.4 & 52.80 & \multirow[t]{2}{*}{$7.0 \times 8.1$} & 25 & 5 & 390 \\
\hline & 17.5 & 17.00 & & 25 & 5 & 590 \\
\hline & 22.3 & 4.80 & \multirow[t]{2}{*}{$9.0 \times 10.6$} & 25 & 5 & 510 \\
\hline & 25.0 & 0.32 & & 25 & 70 & 500 \\
\hline \multirow{4}{*}{$\begin{array}{l}\mathbf{C} \\
6 \mathrm{CdTe}\end{array}$} & 12.4 & 46.8 & \multirow[t]{2}{*}{$7.9 \times 9.1$} & 20 & 5 & 400 \\
\hline & 17.5 & 18.0 & & 20 & 5 & 570 \\
\hline & 22.3 & 4.52 & \multirow[t]{2}{*}{$9.0 \times 10.7$} & 20 & 5 & 590 \\
\hline & 25.0 & 0.32 & & 20 & 70 & 530 \\
\hline \multirow{4}{*}{$\begin{array}{l}\text { D } \\
5 \mathrm{CdTe}\end{array}$} & 22.3 & 5.06 & \multirow[t]{4}{*}{$9.0 \times 10.7$} & 25 & 5 & 580 \\
\hline & 25 & 0.31 & & 25 & 70 & 510 \\
\hline & 26 & 0.26 & & 25 & 90 & 470 \\
\hline & 27 & 0.04 & & 25 & 700 & 500 \\
\hline \multirow{3}{*}{$\begin{array}{l}\mathbf{E} \\
5 \mathrm{CdTe}\end{array}$} & 17.5 & 18.0 & $7.9 \times 9.1$ & 30 & 8 & 840 \\
\hline & 22.3 & 4.52 & \multirow[t]{2}{*}{$9.0 \times 10.7$} & 30 & 8 & 840 \\
\hline & 25 & 0.32 & & 30 & 110 & 700 \\
\hline
\end{tabular}

Table S1: Overview of all diffraction data collected. A data series refers to a set of four datasets collected at four different energies from a single position on a crystal. Doses were calculated using $R A D D O S E-3 D$ taking photoelectron escape into account. 
bioRx|v preprint doi: https://doi.org/10.1101/2021.01.21.427633; this version posted January 21, 2021. The copyright holder for this preprint (which was not certified by peer review) is the author/funder, who has granted bioRxiv a license to dişlay the preprint in

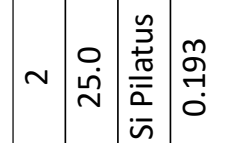

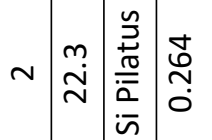

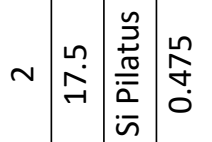

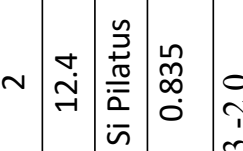

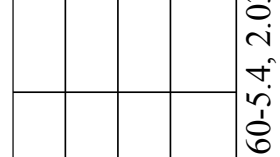

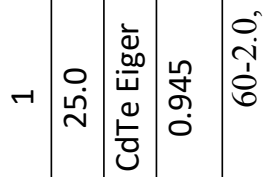

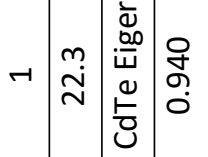

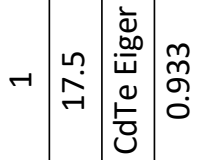

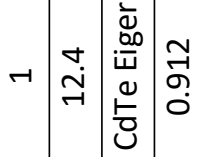

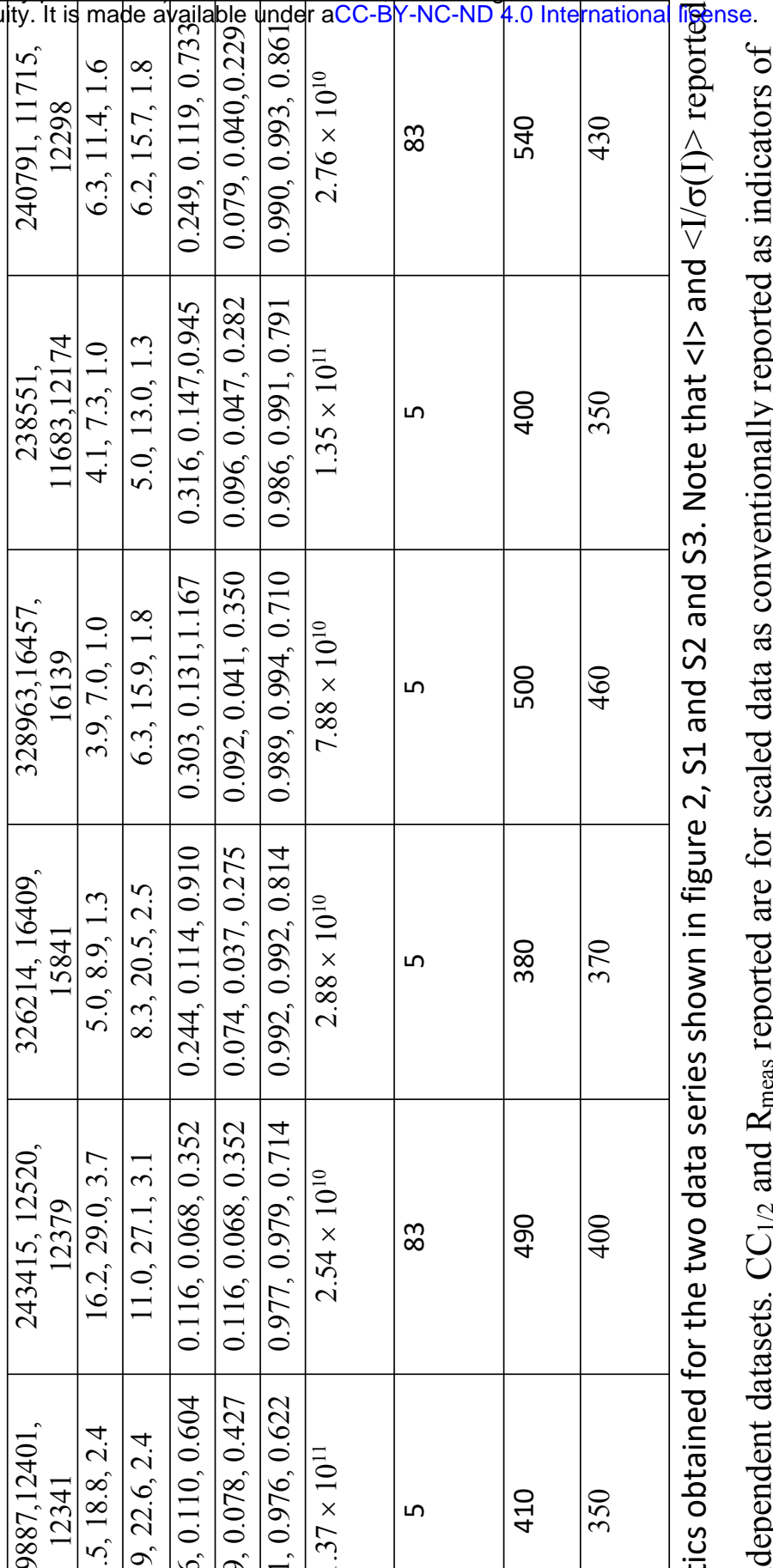

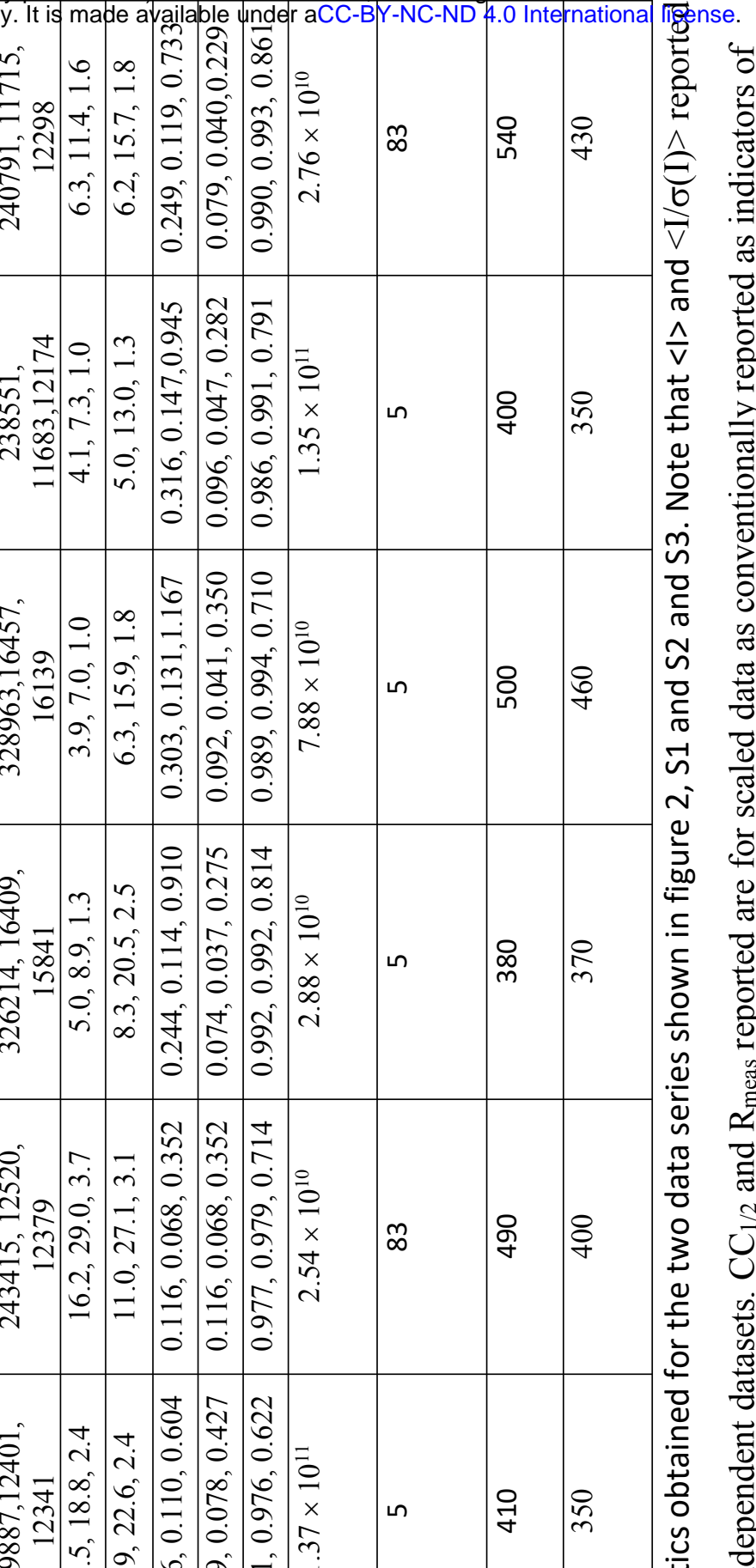

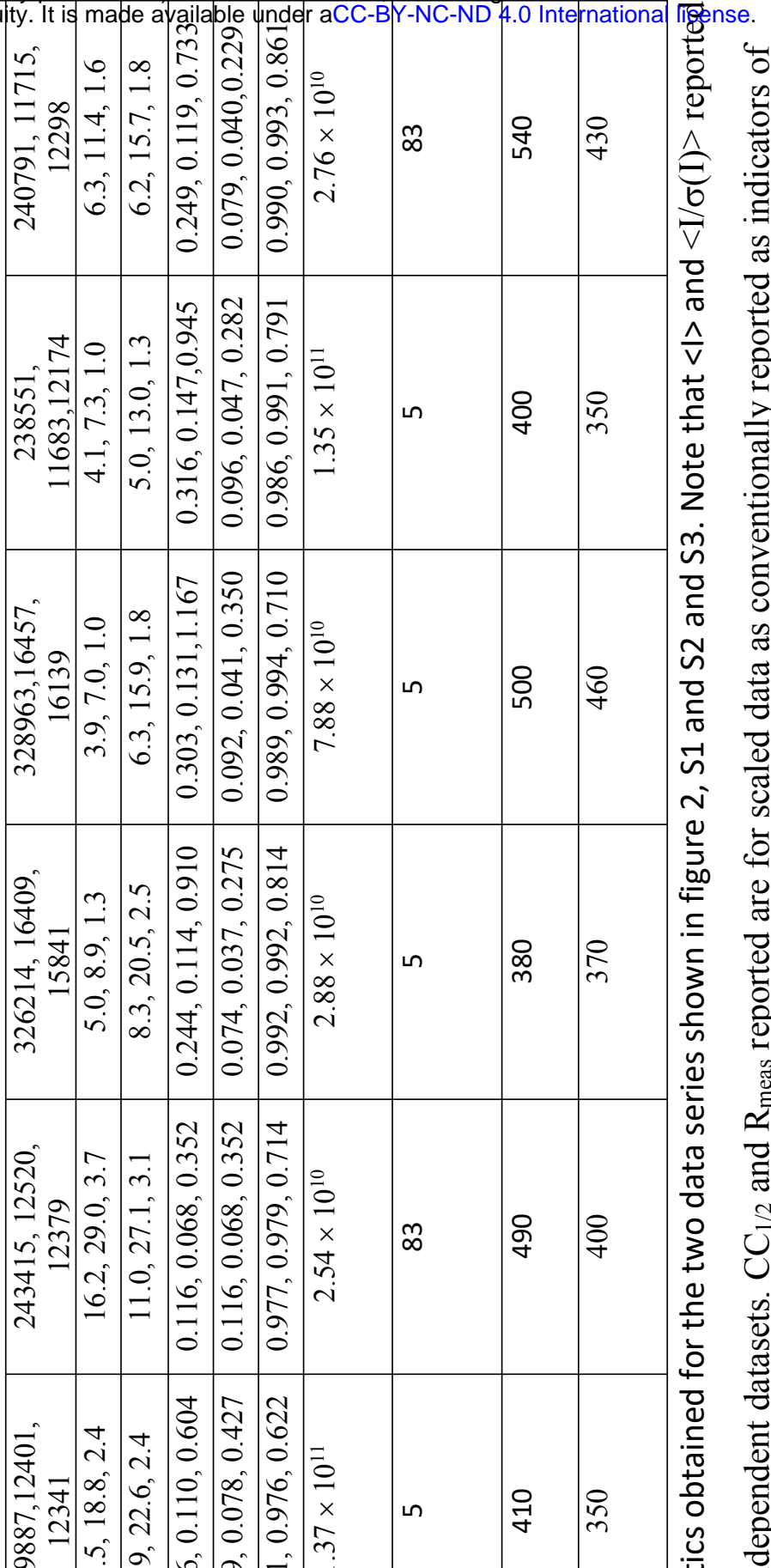

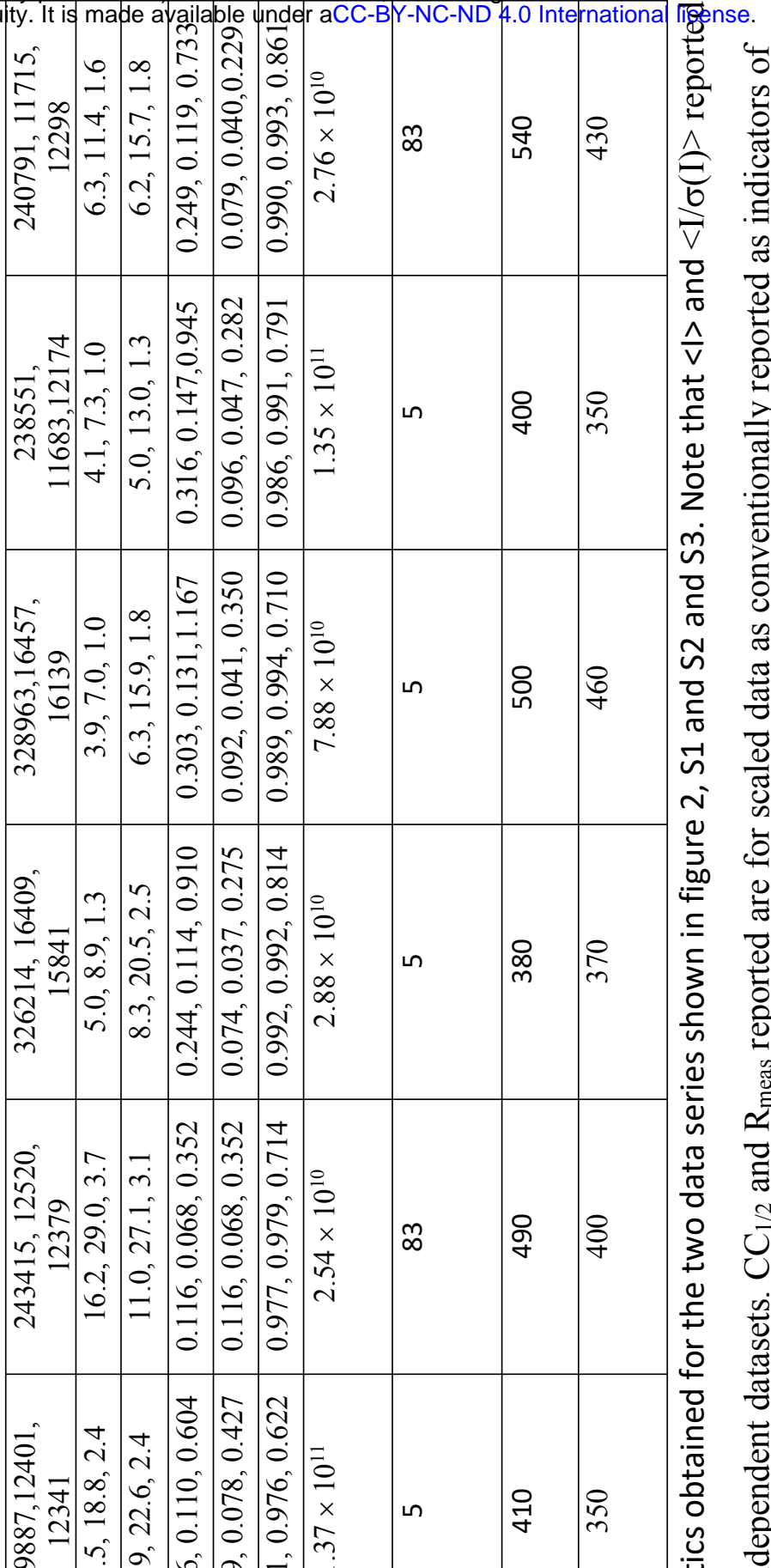

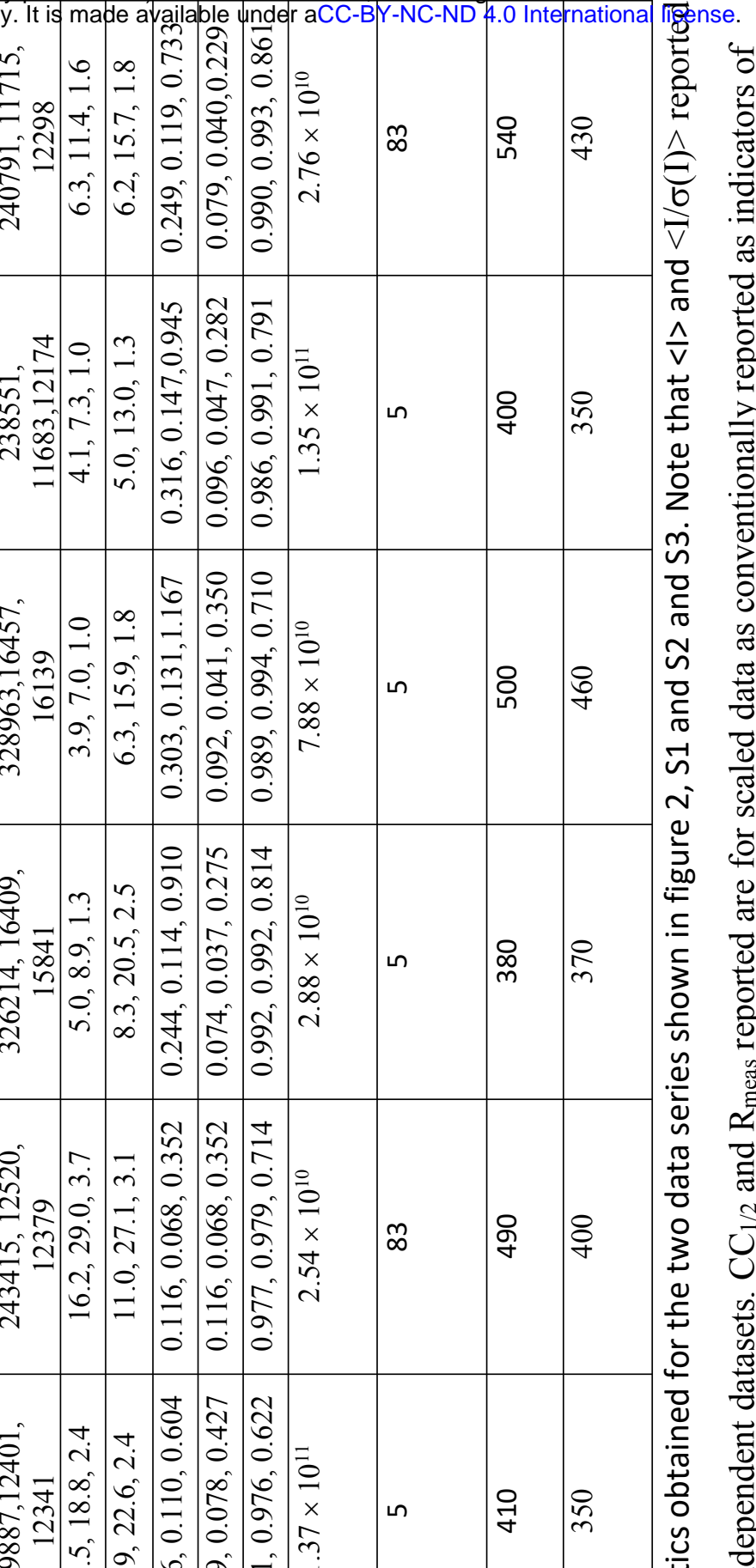

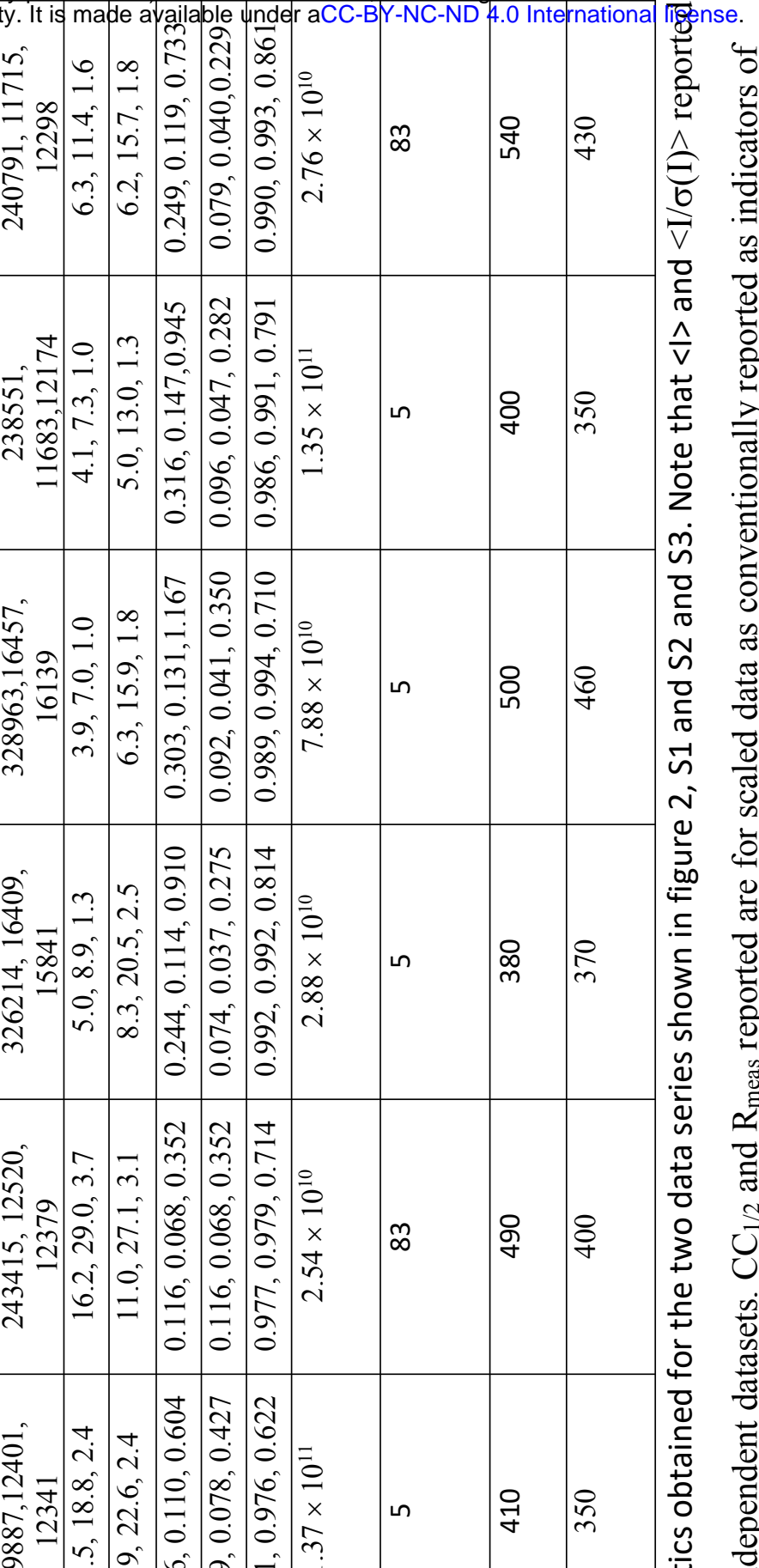

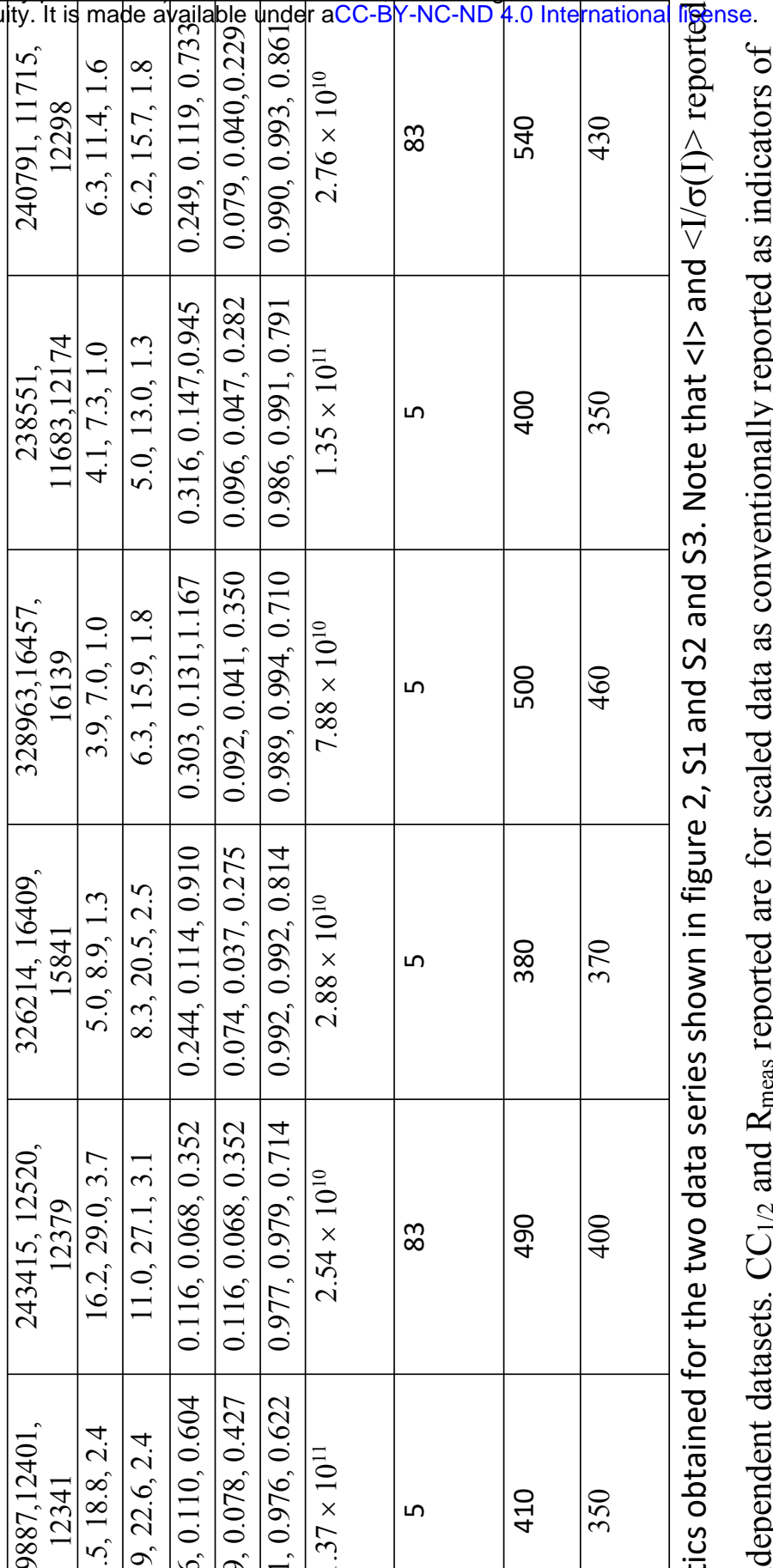

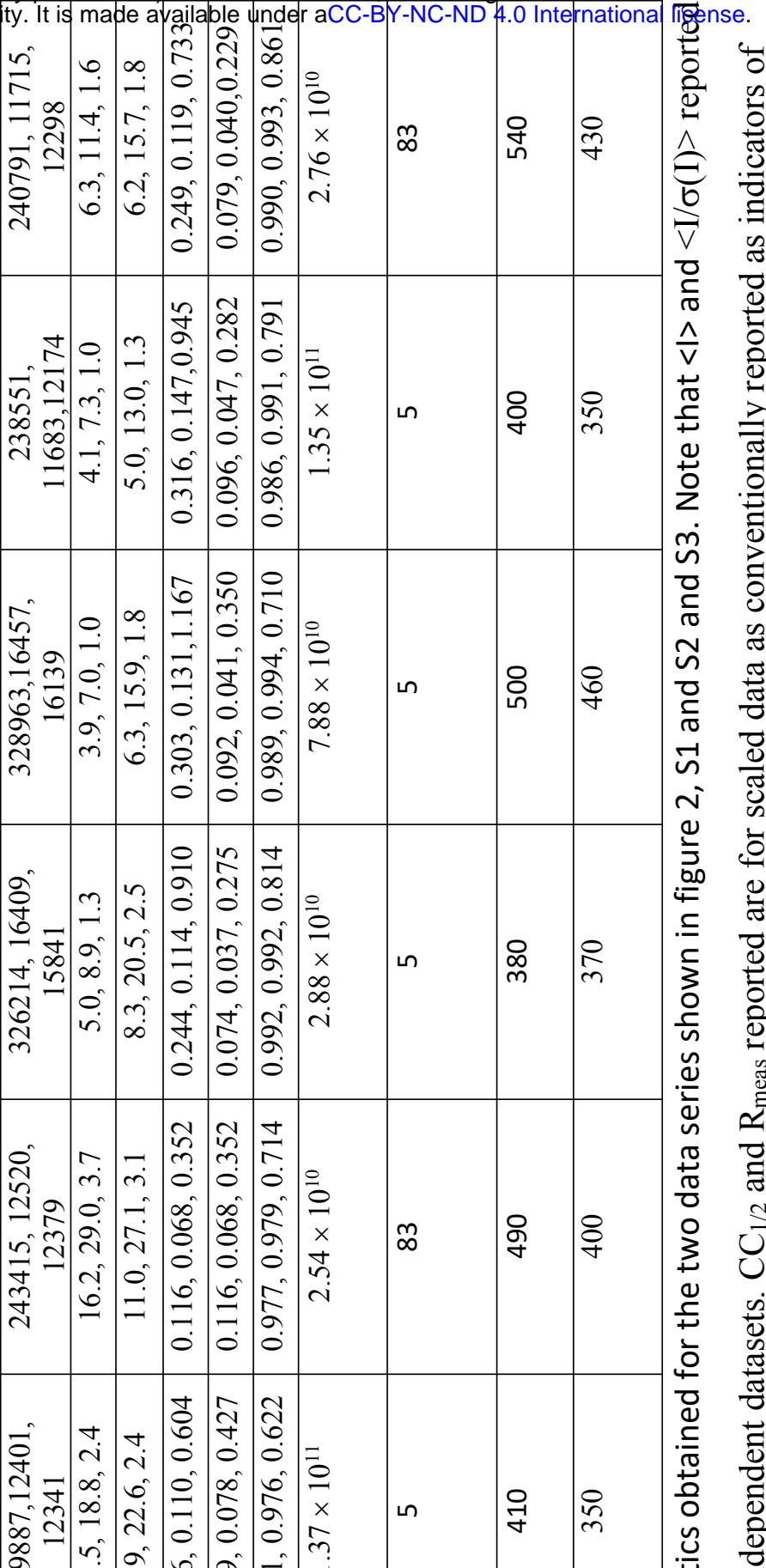

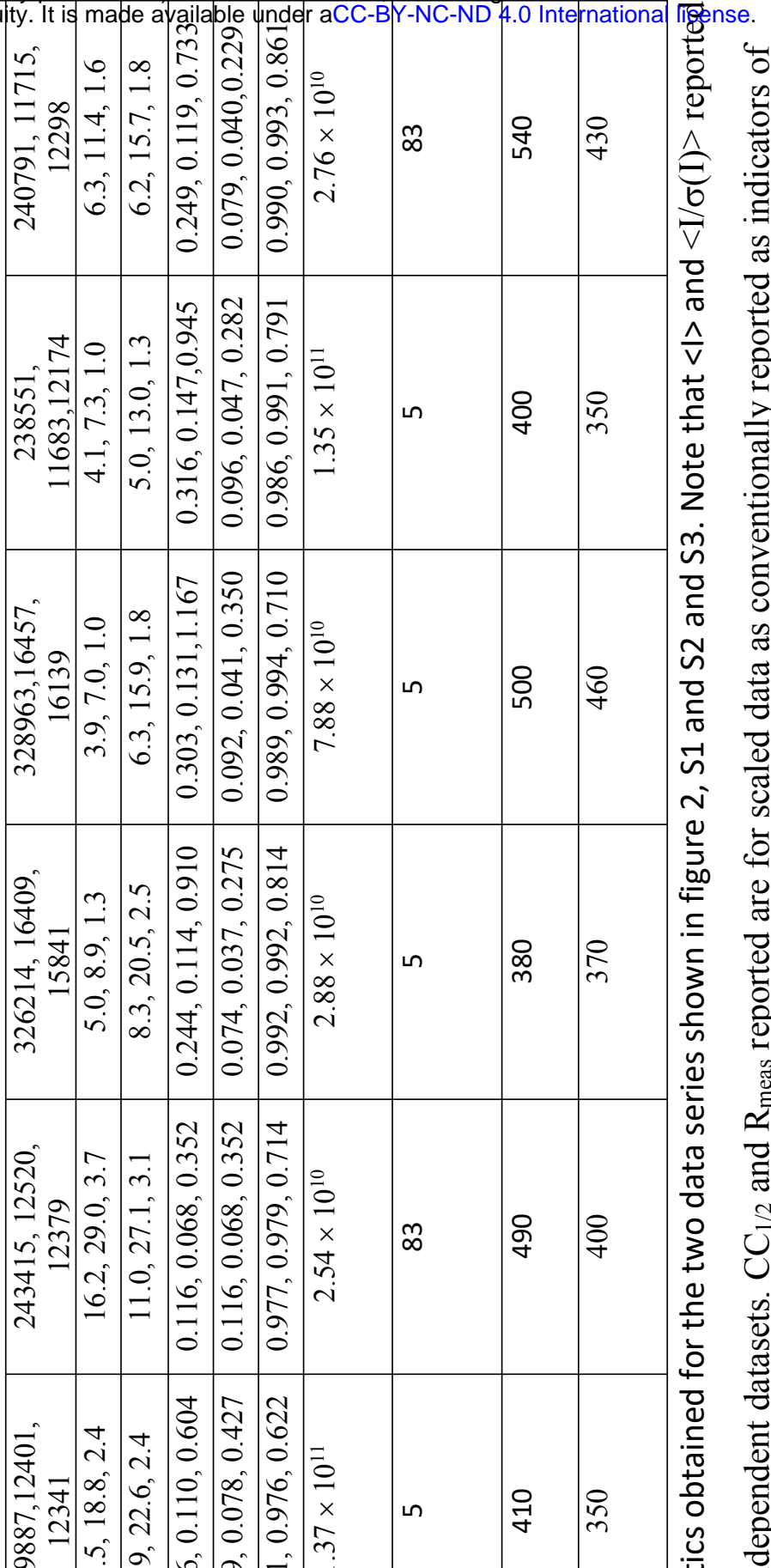

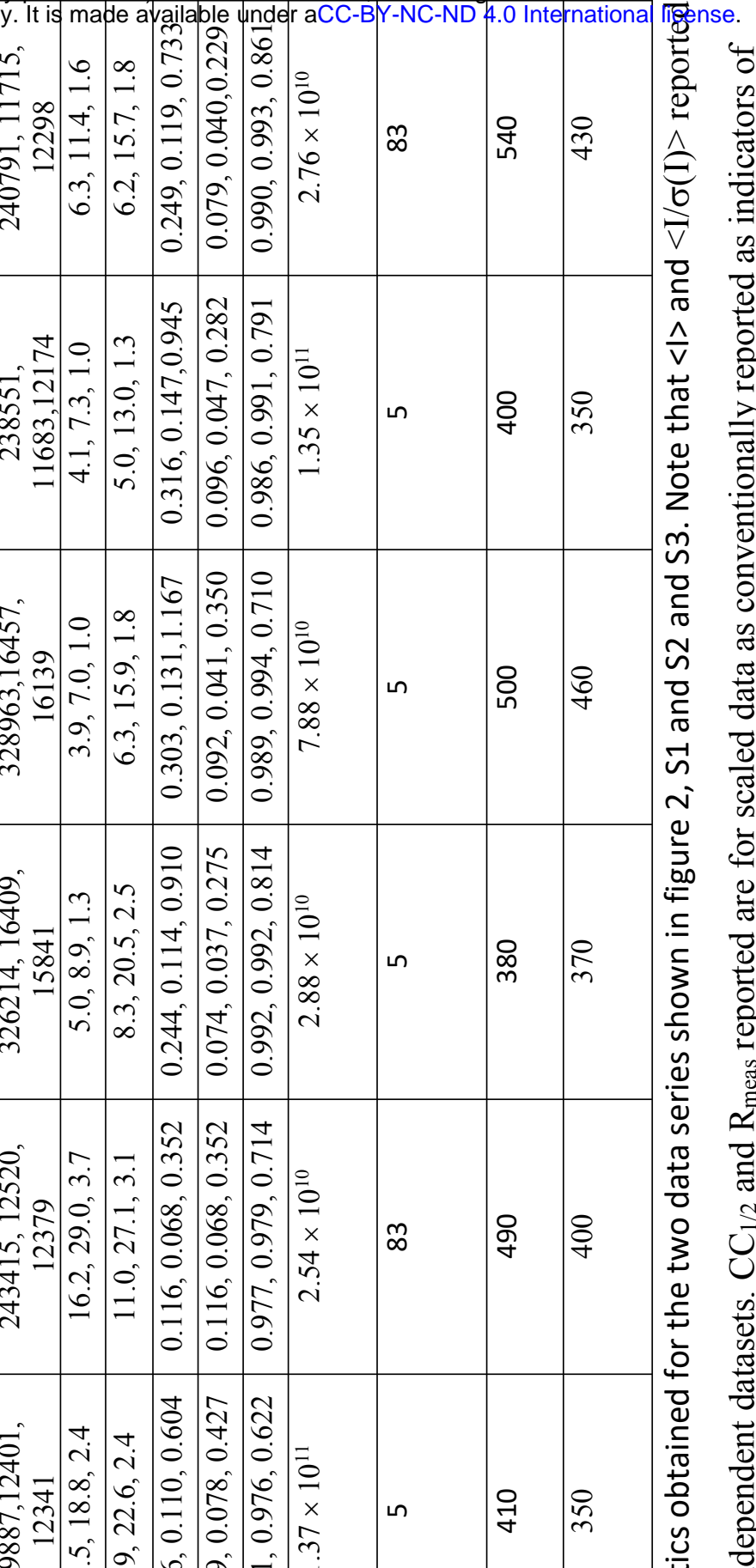

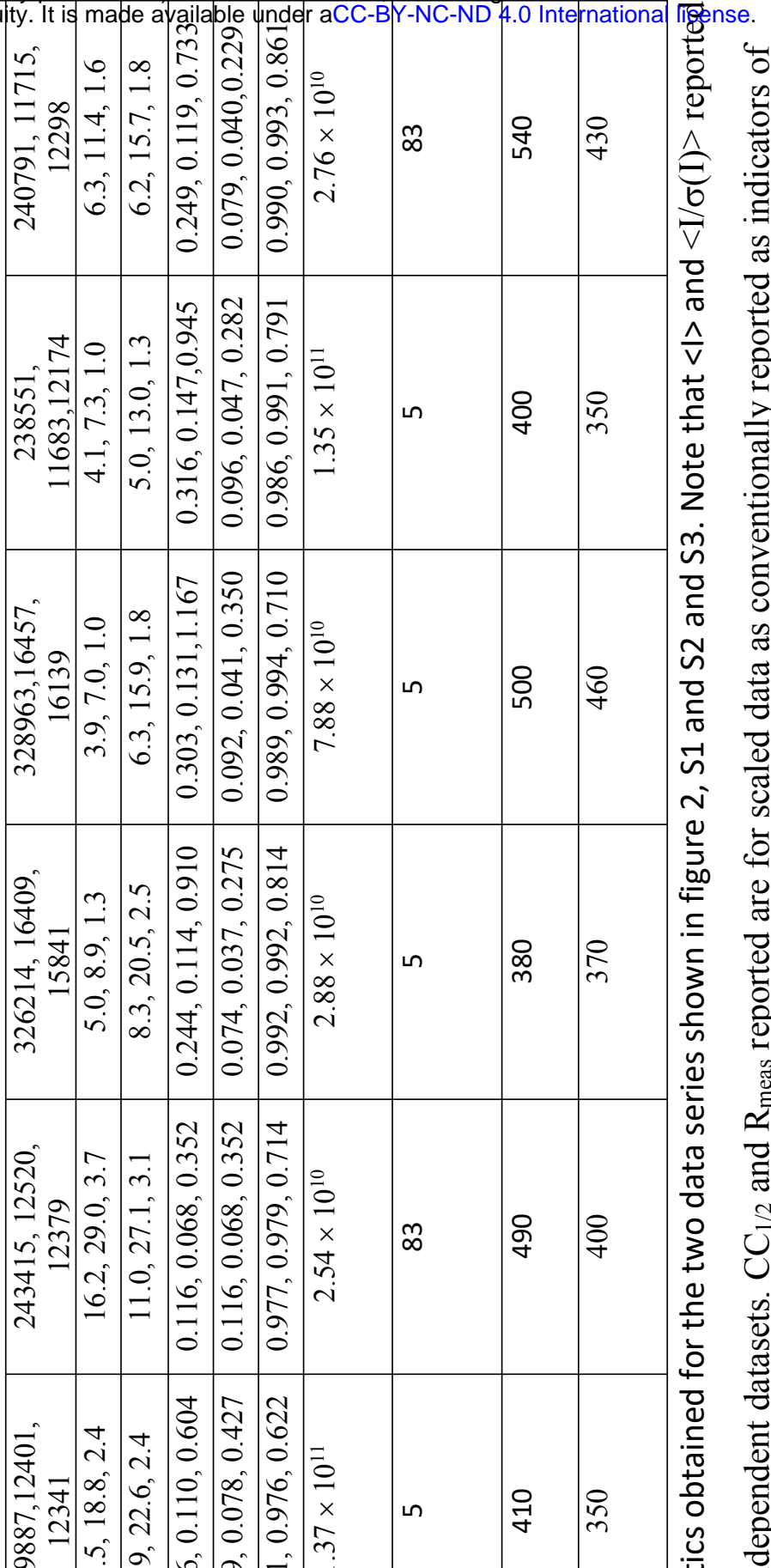

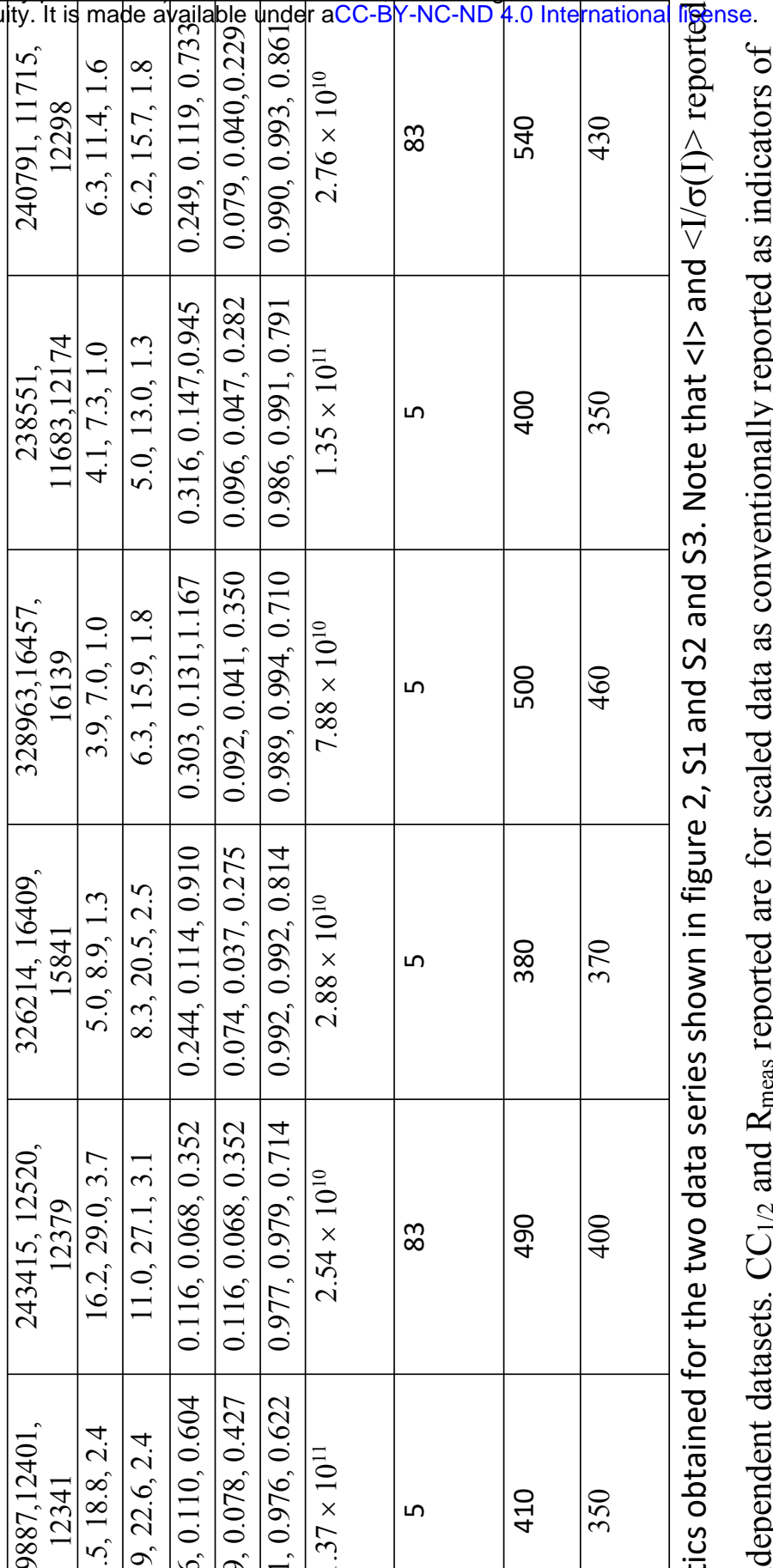

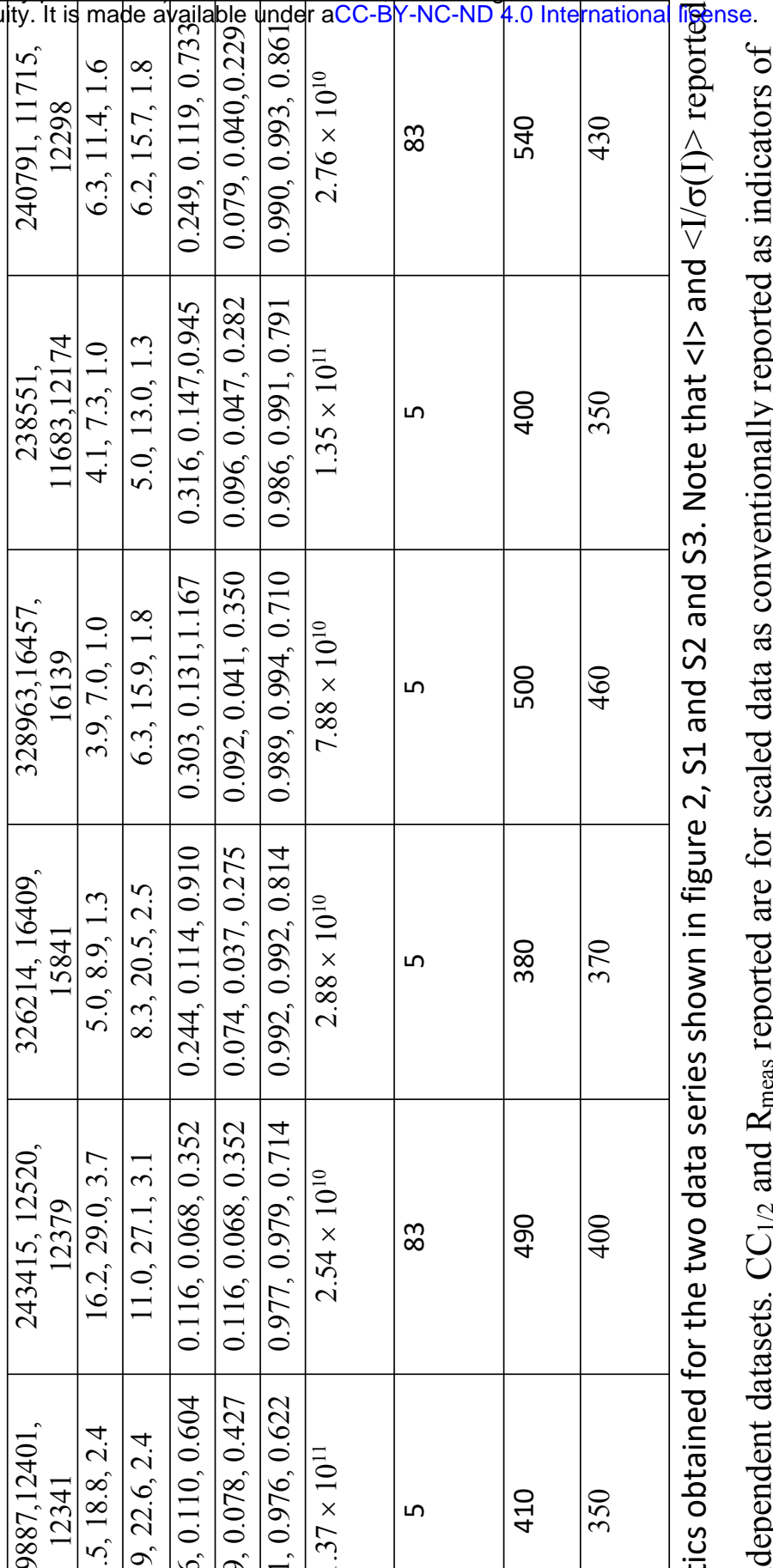

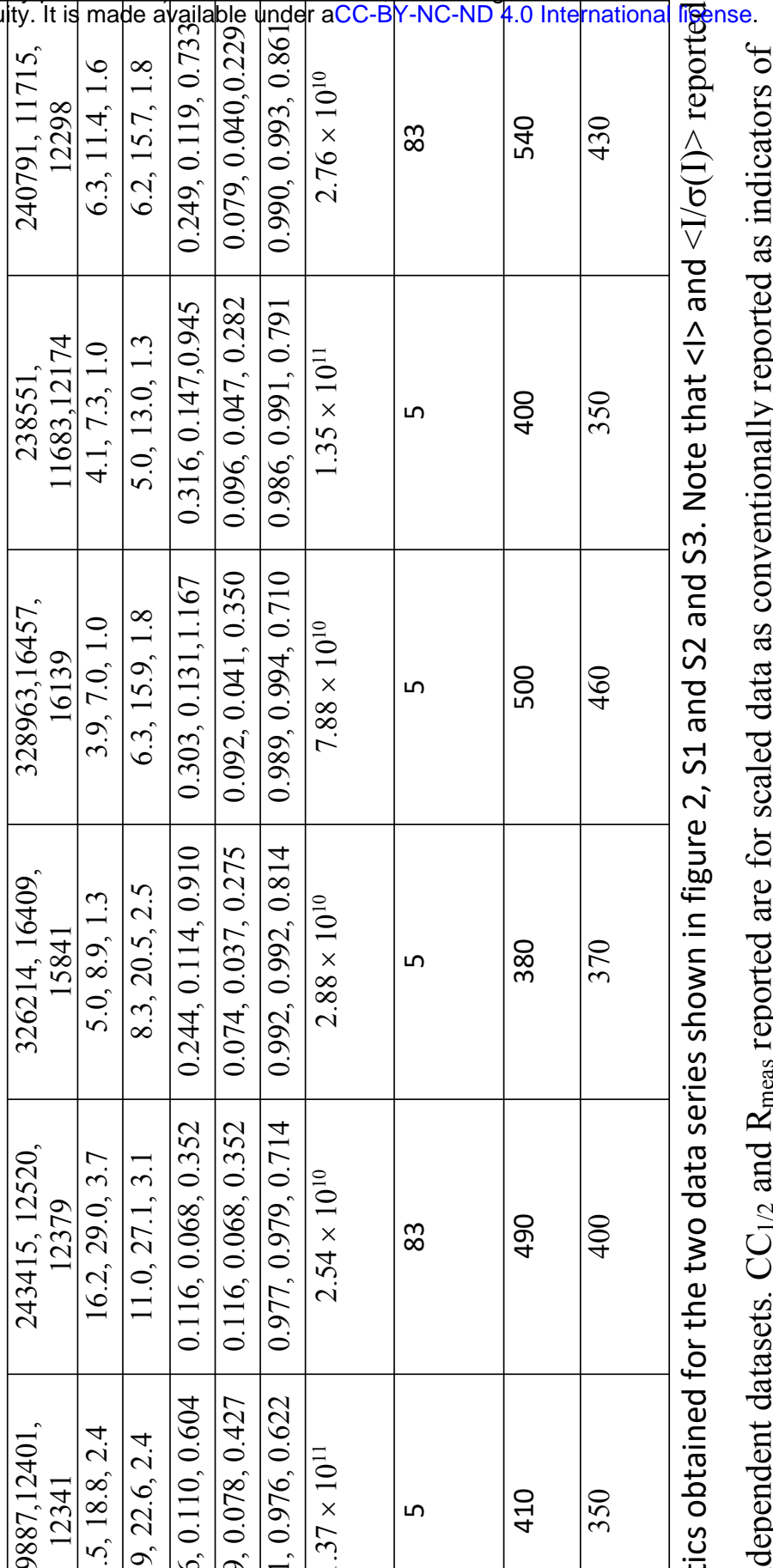

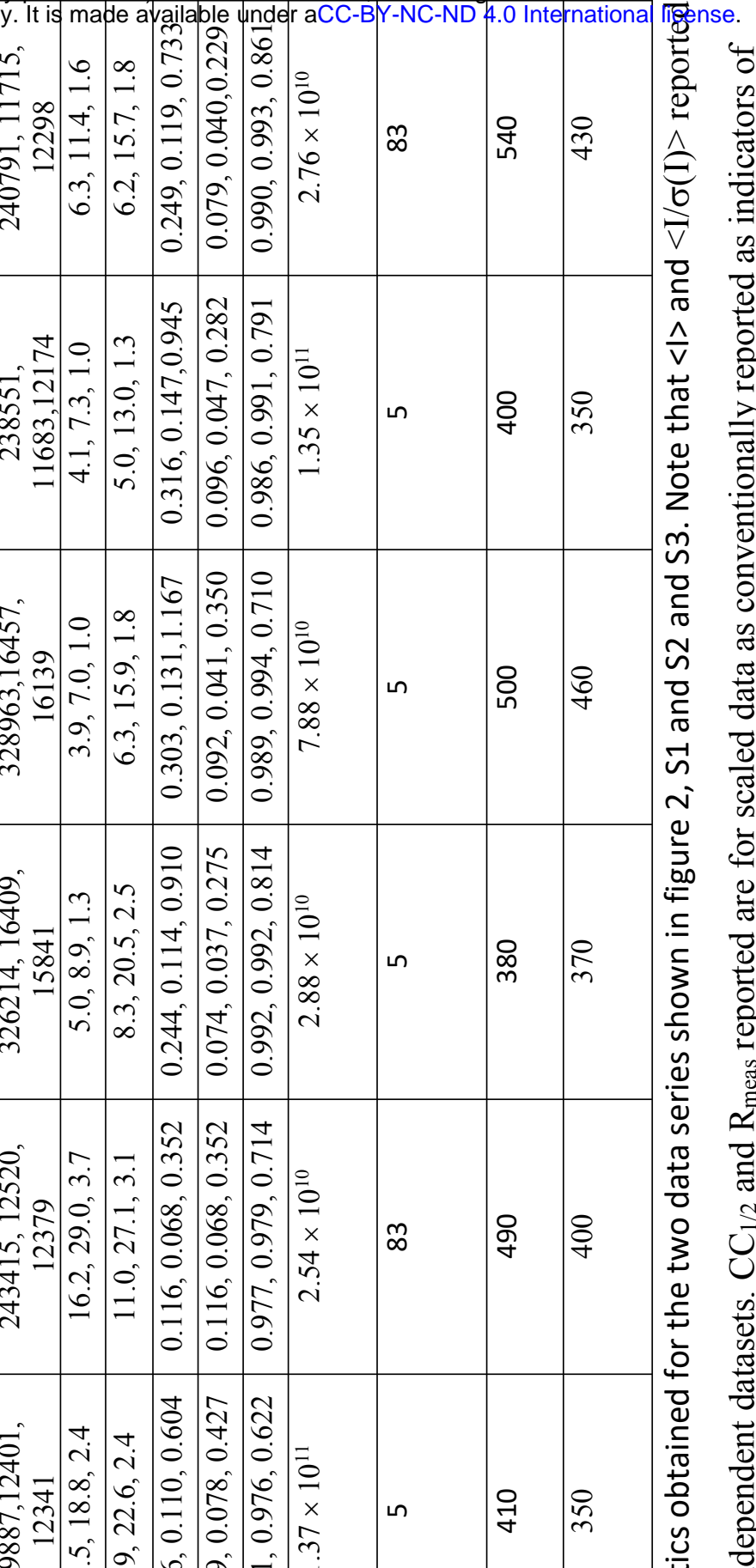

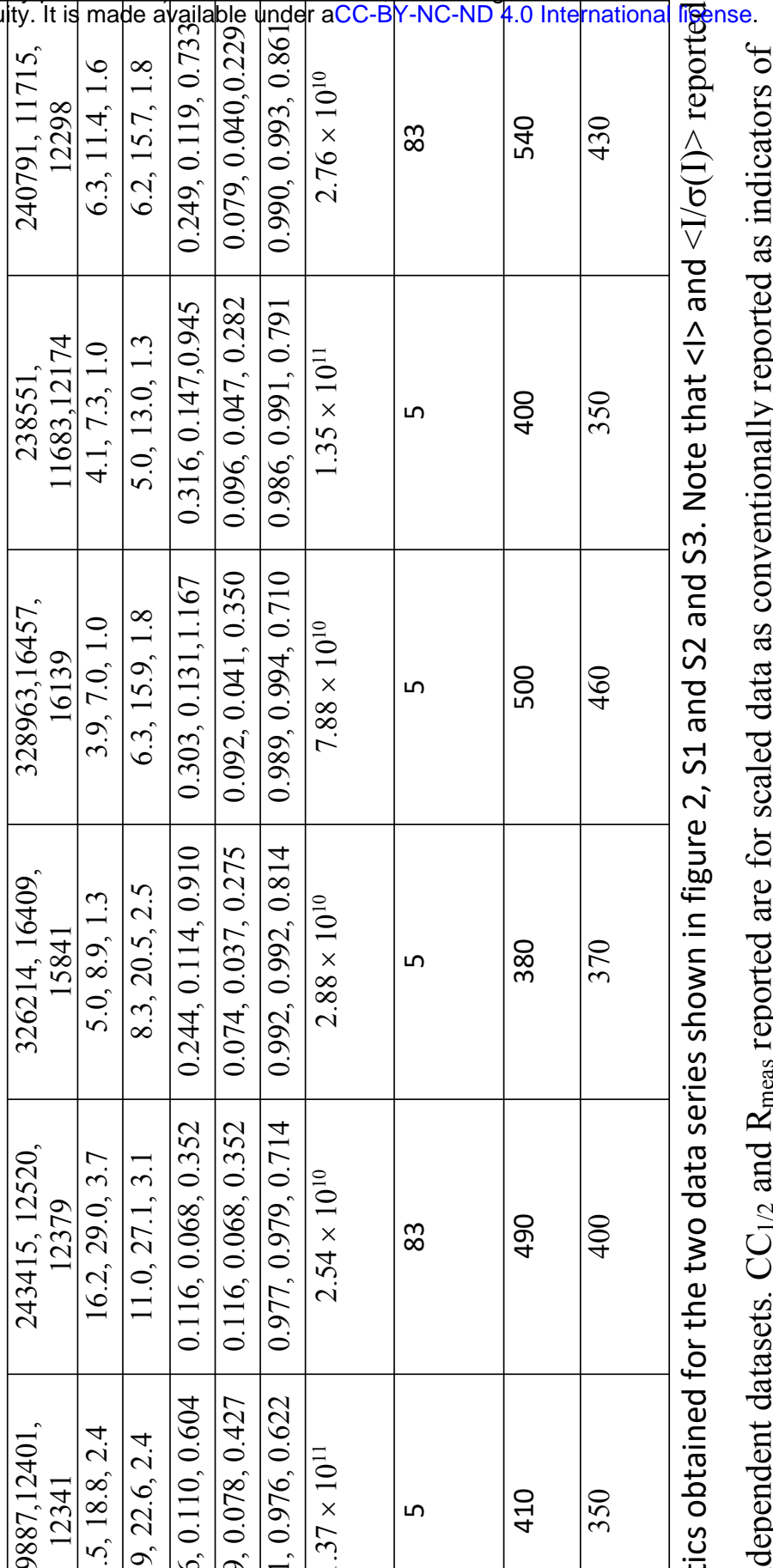

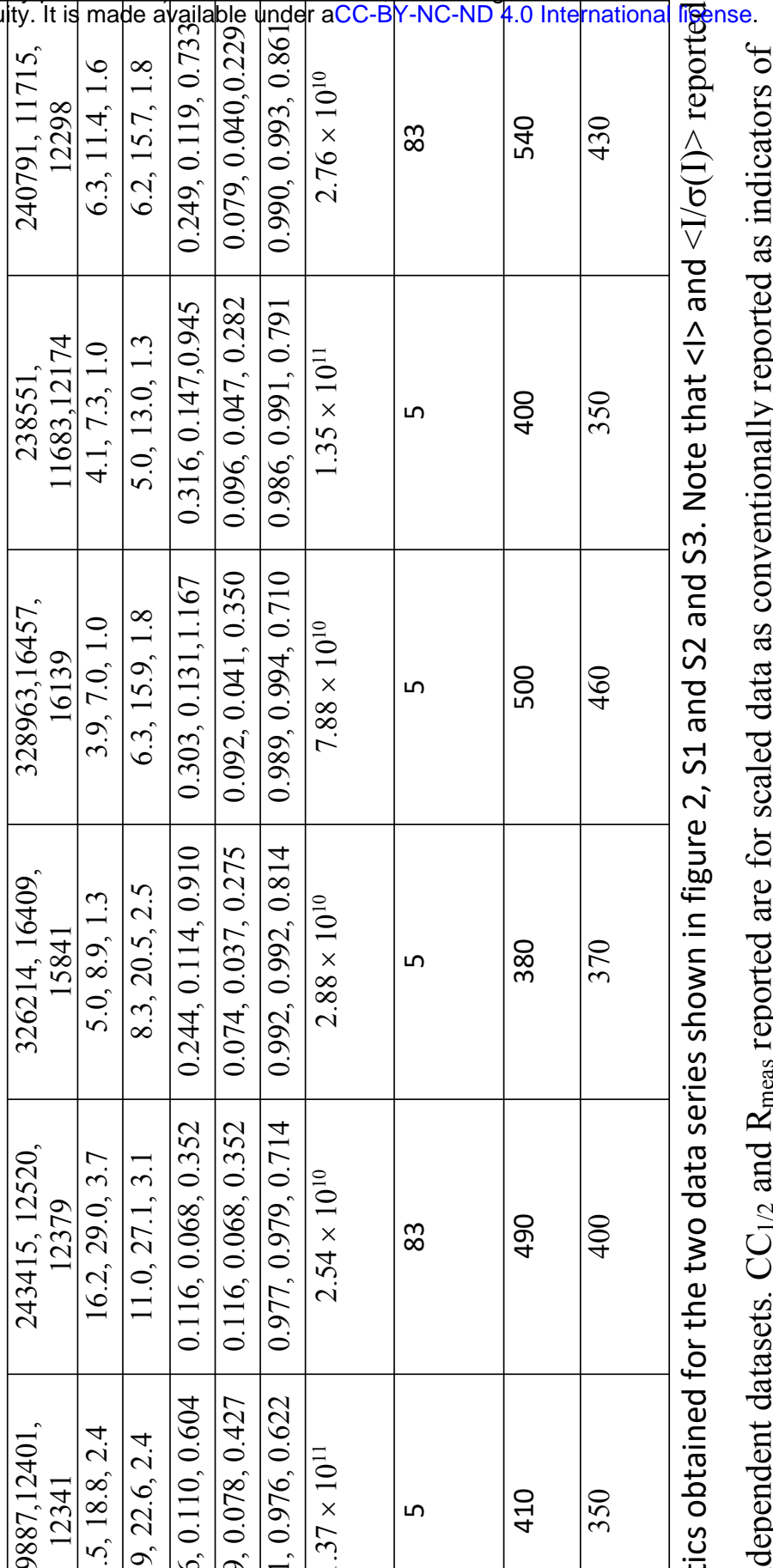

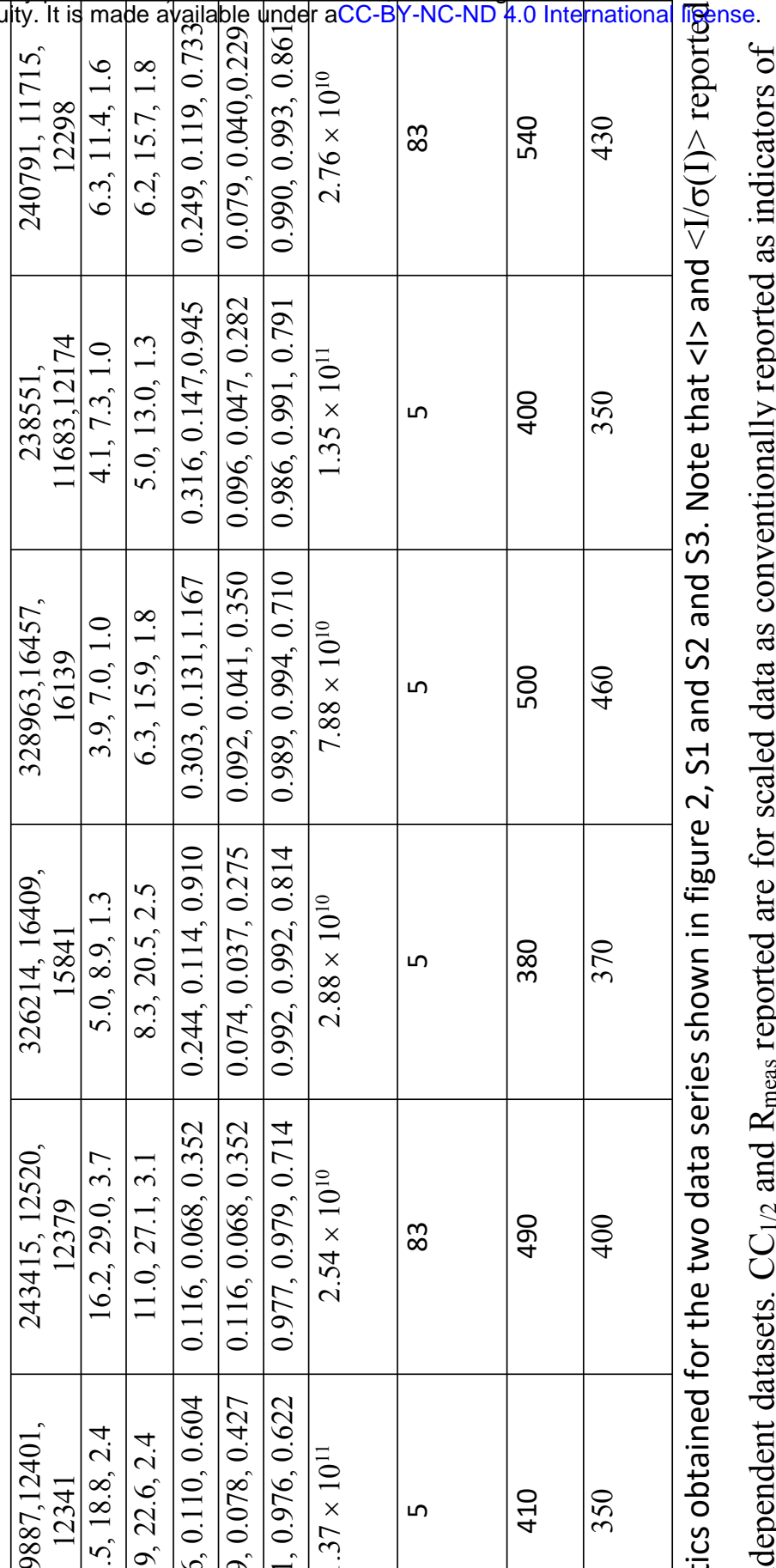

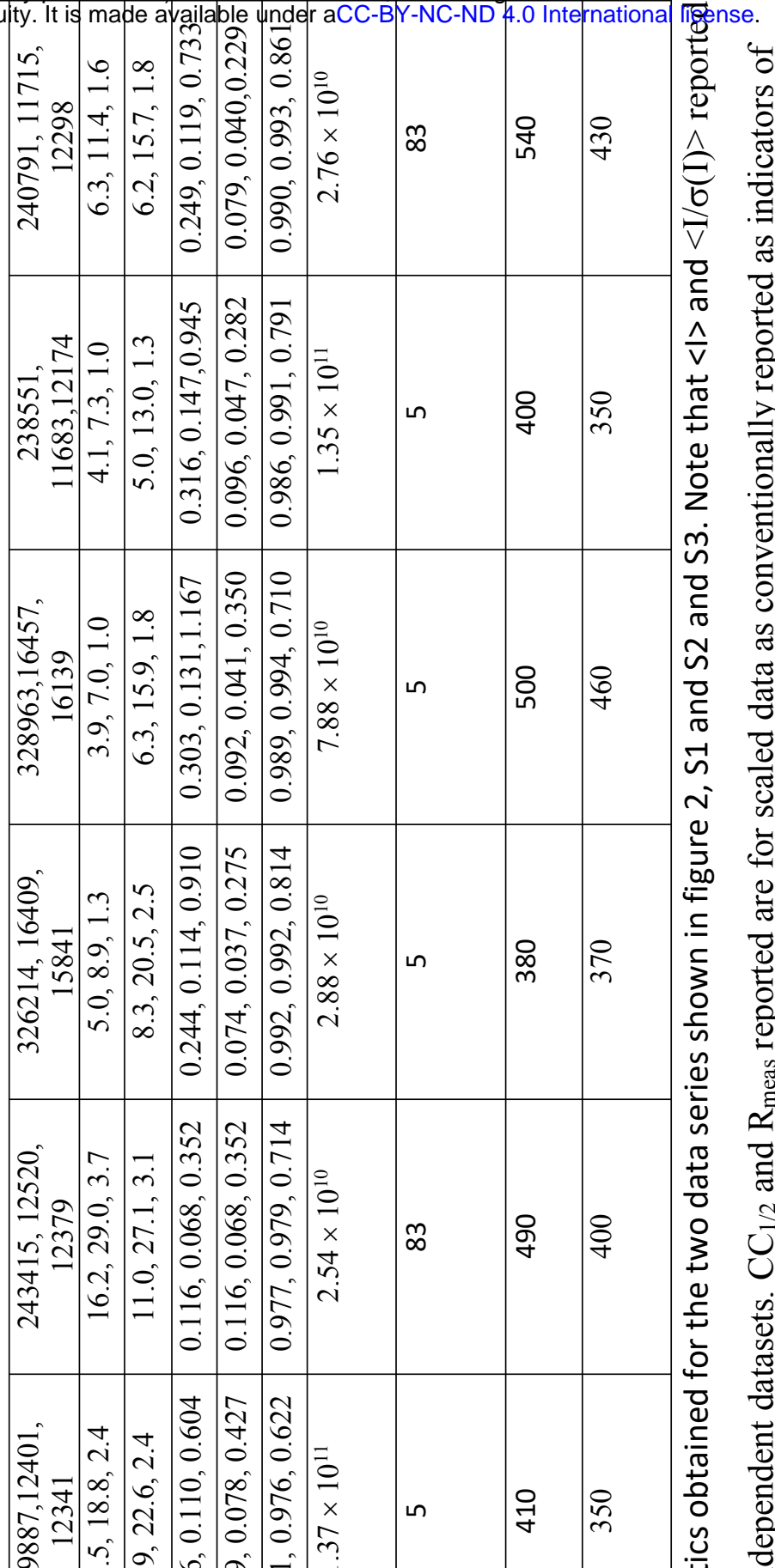

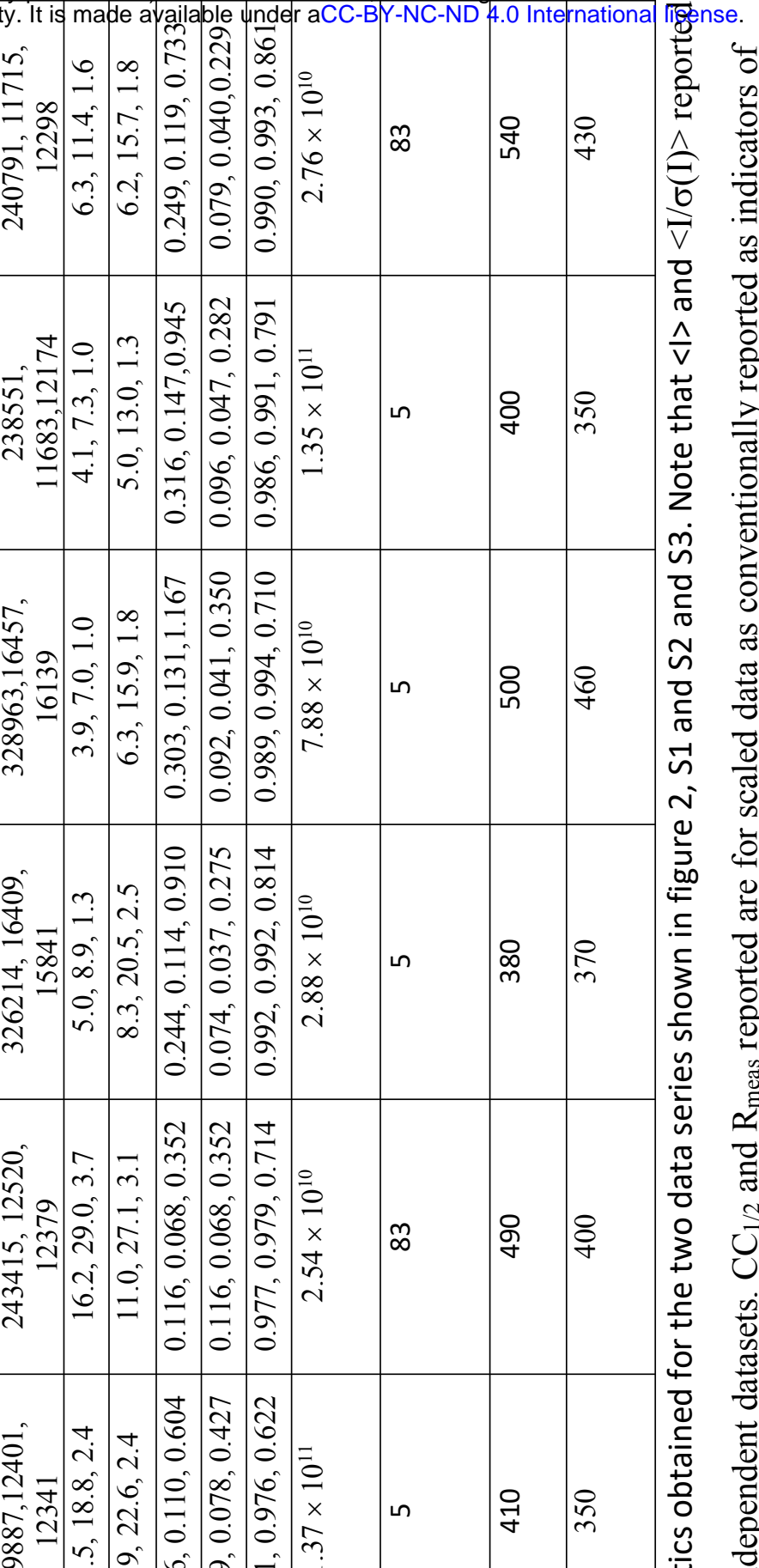

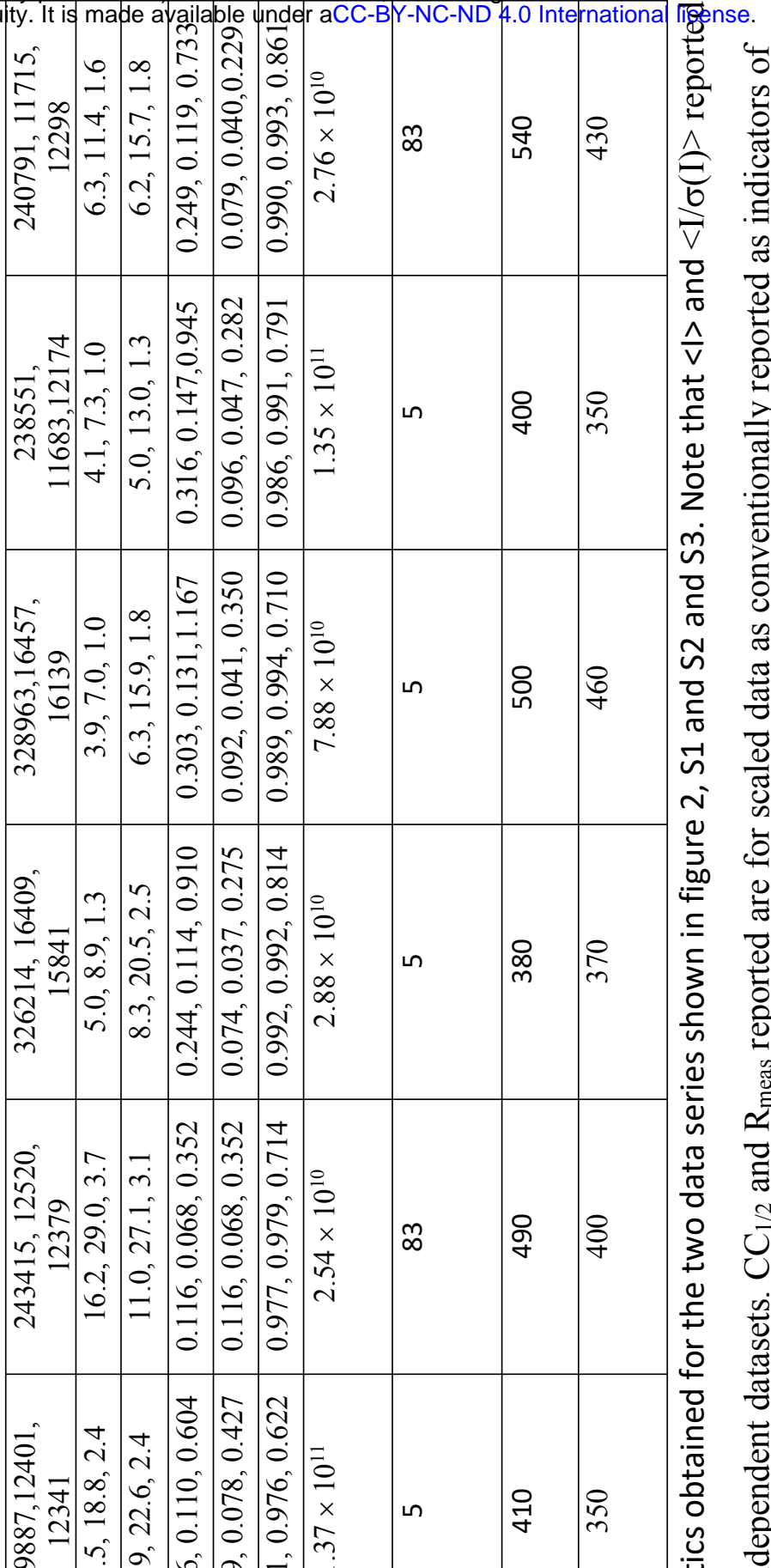

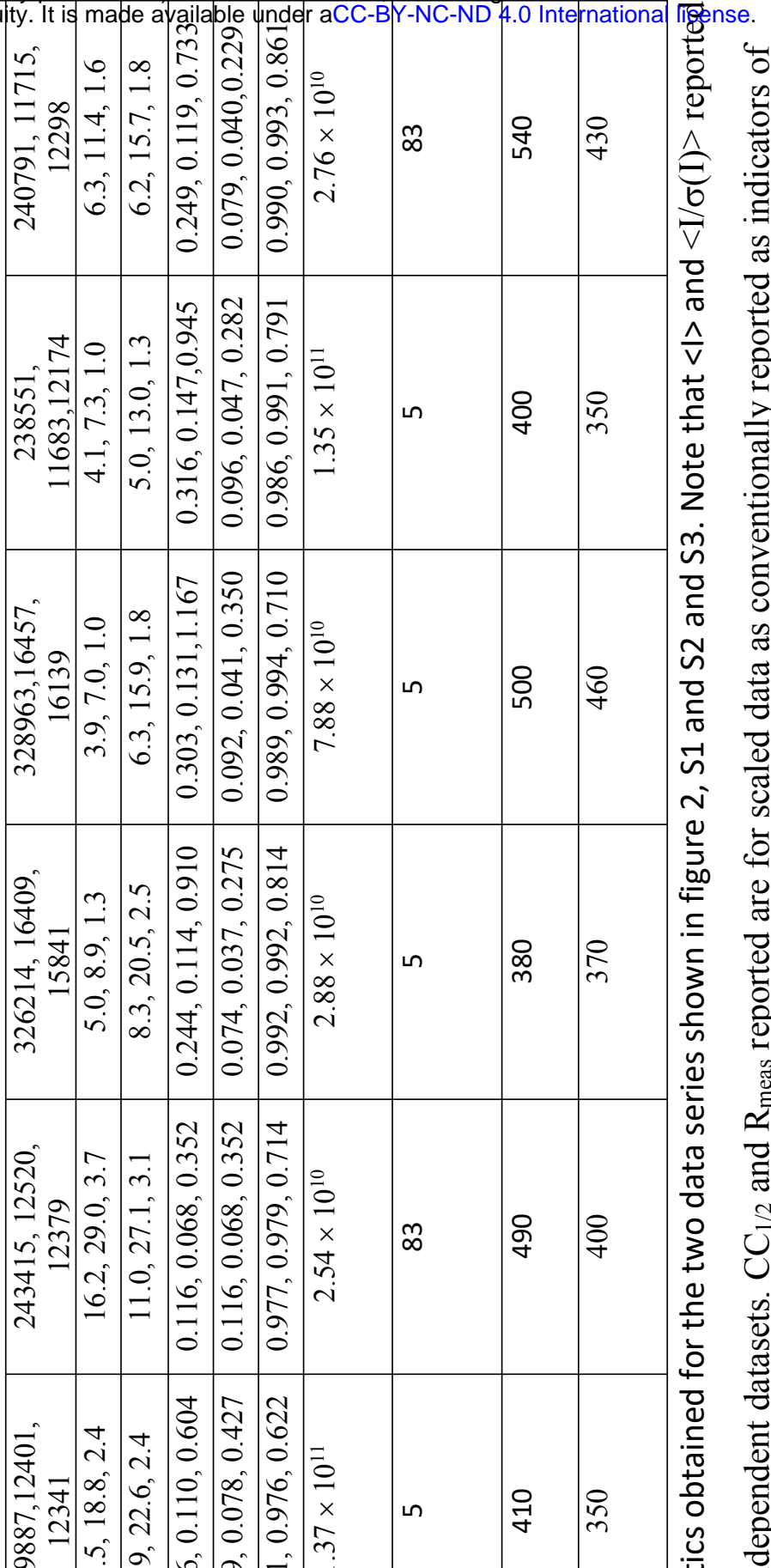

ते $\cong$ ஸे

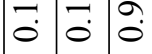

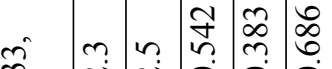

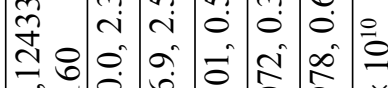

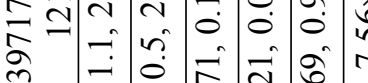

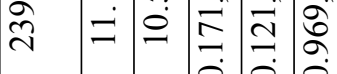

$\infty \quad$ a l a

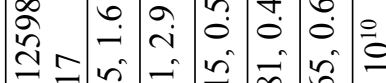

클

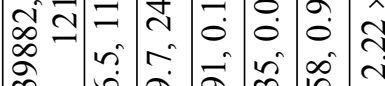

ते 


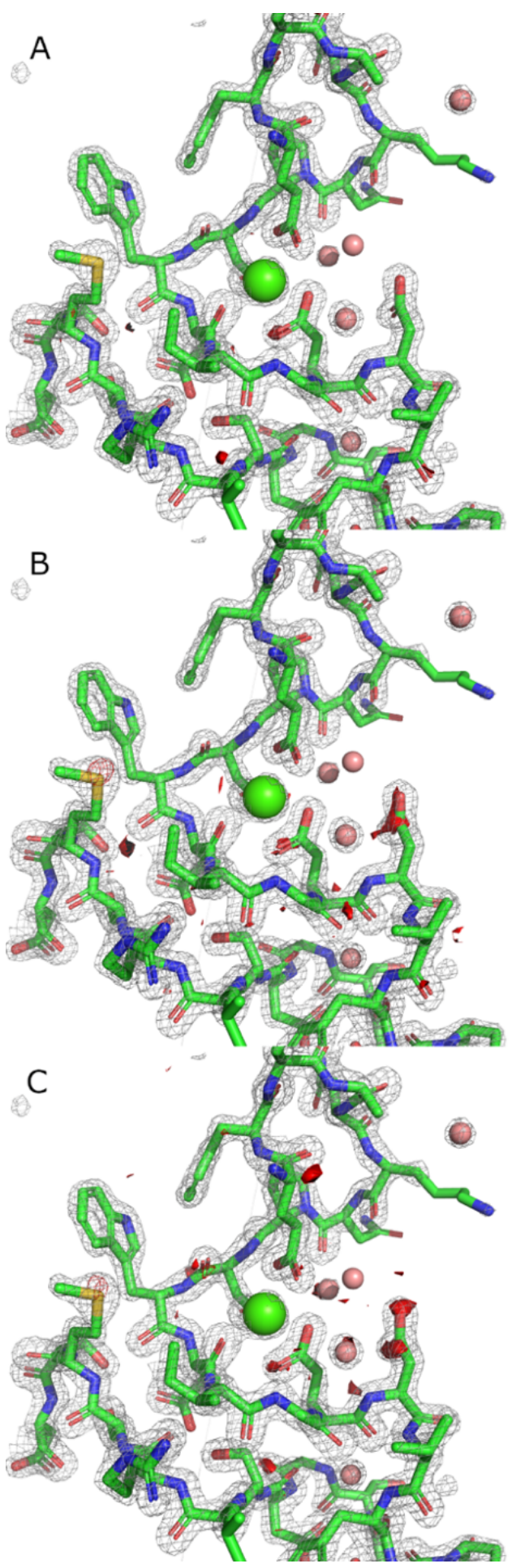

Figure. S1: Exemplar electron density obtained from sequential datasets forming a data series. The grey mesh represents the $2 \mathrm{Fo}-\mathrm{Fc}$ map and the stick model the refined thermolysin structure from the first dataset in the series $(17500 \mathrm{eV})$. Data were recorded using the CdTe Eiger detector and processed using phenix.refine (Liebschner et al. (2019)). These data were collected in the 
bioRxiv preprint doi: https://doi.org/10.1101/2021.01.21.427633; this version posted January 21, 2021. The copyright holder for this preprint (which was not certified by peer review) is the author/funder, who has granted bioRxiv a license to display the preprint in perpetuity. It is made available under aCC-BY-NC-ND 4.0 International license.

order: $17500 \mathrm{eV}, 22300 \mathrm{eV}, 25000 \mathrm{eV}, 12400 \mathrm{eV}$. In A, B, and C Fo-Fo difference maps have been superimposed onto the refined data, shown in opaque surface representation and contoured to 4 sigma. A shows $12400 \mathrm{eV}$ minus $17500 \mathrm{eV}$, B shows $12400 \mathrm{eV}$ minus $22300 \mathrm{eV}$, C shows $12400 \mathrm{eV}$ minus $25000 \mathrm{eV}$. Maps produced with phenix.fobs_minus_fobs_map. Very slight density loss is visible on acidic residues ASP190, ASP191 and the sulphur atom of MET205 in the third and fourth datasets collected.
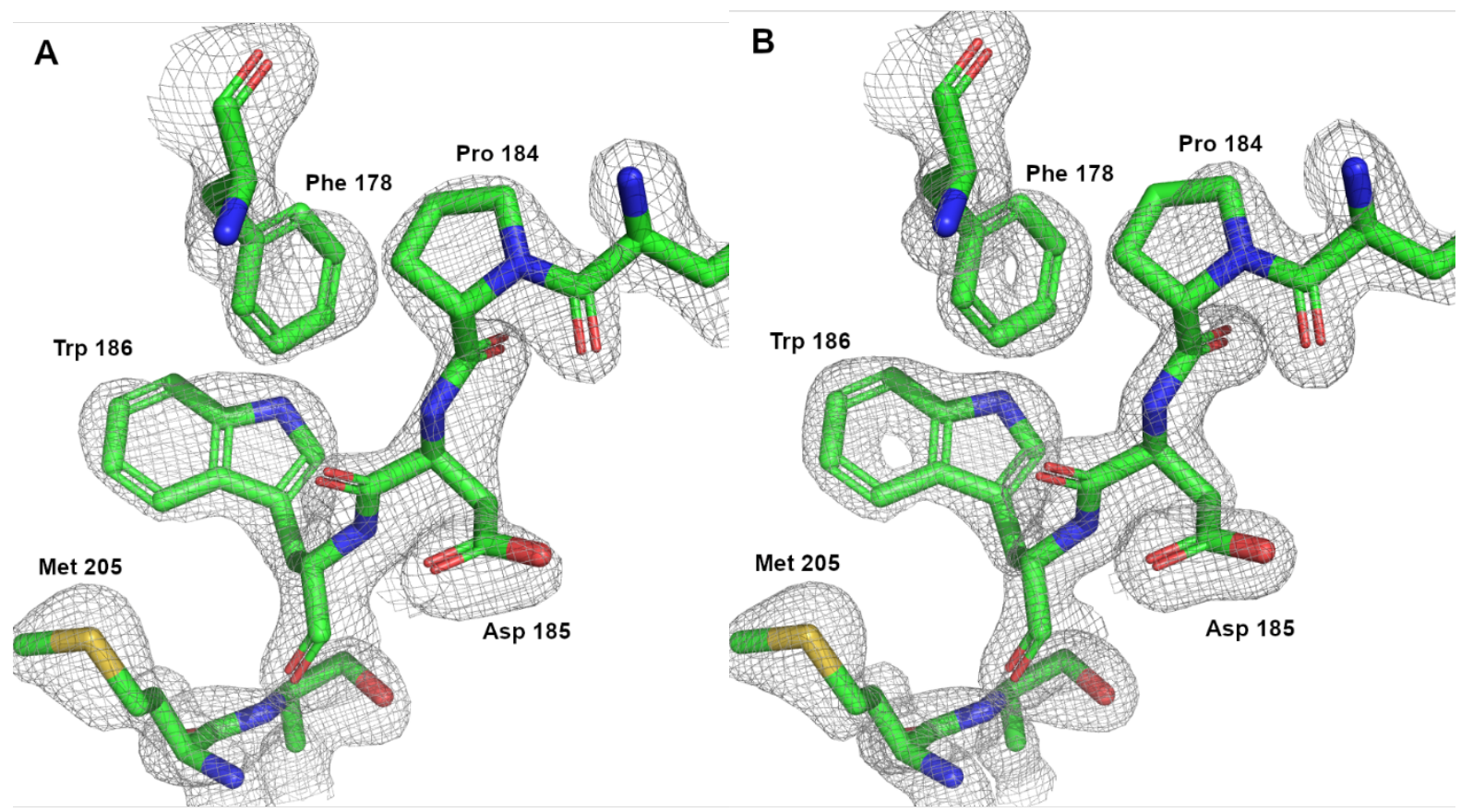

Figure. S2: Composite omit maps illustrating differences between data recorded using the $\mathrm{CdTe}$ detector at different energies but the same total diffracted intensity. (A) Example volume of electron density obtained from 100 degrees of thermolysin data collected at $12.4 \mathrm{keV}$, diffracting to $1.90 \AA$ using a $\mathrm{CC}_{1 / 2}>0.3$ threshold. (B) electron density of the same volume obtained by collecting from the same position of the crystal at $25 \mathrm{keV}$ and diffracting to $1.61 \AA$ using the same critereon. The increased resolution is apparent in the electron density of rings and clarity of other features. Maps contoured to $1.5 \sigma$ in both cases. Models were refined and maps obtained using Phenix; R/R free $0.210 / 0.245$ in the case of (A) and $R / R_{\text {free }} 0.202 / 0.229$ in the case of (B). 
bioRxiv preprint doi: https://doi.org/10.1101/2021.01.21.427633; this version posted January $21,2021$. The copyright holder for this preprint (which was not certified by peer review) is the author/funder, who has granted bioRxiv a license to display the preprint in perpetuity. It is made available under aCC-BY-NC-ND 4.0 International license.

Figure created using Pymol (The PyMOL Molecular Graphics System, Version 2.0 Schrödinger, LLC).

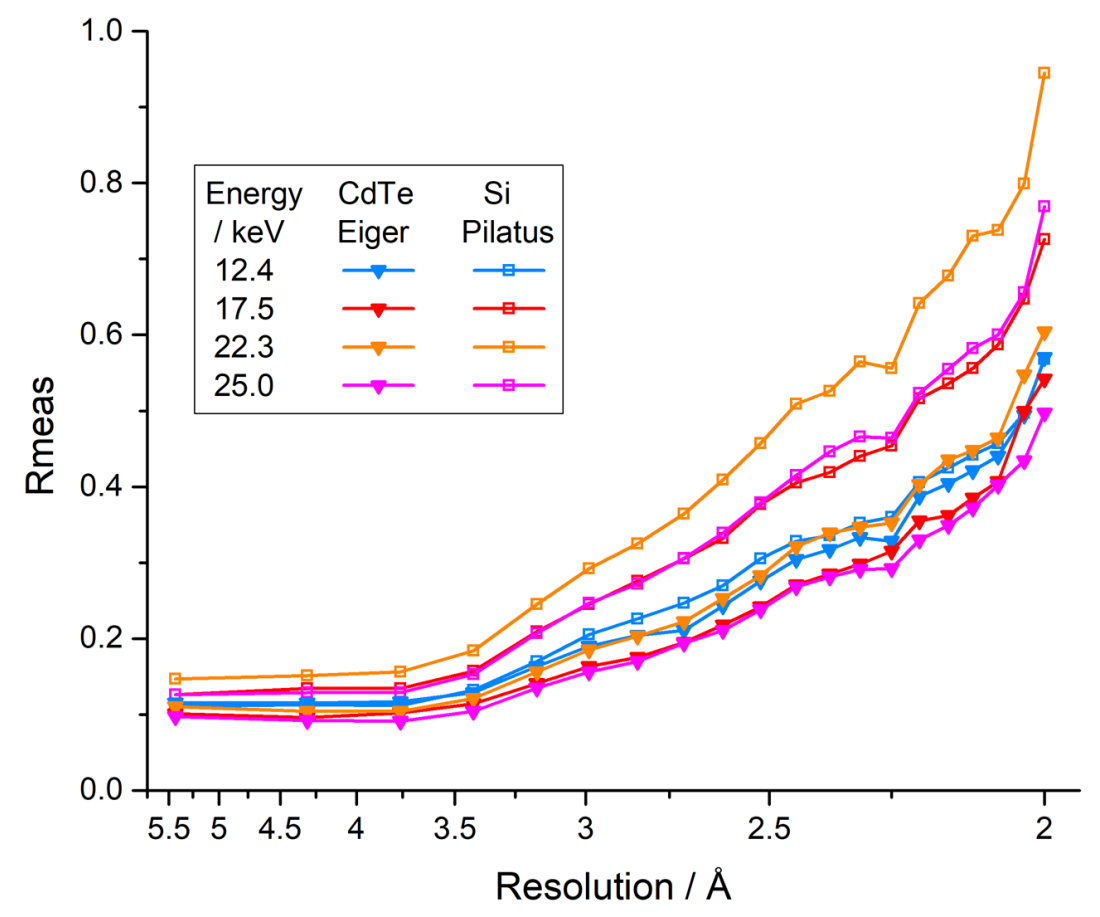

Figure S3: Redundancy independent merging $\mathrm{R}$ value $\mathrm{R}_{\text {meas }}$ for the data series summarized in figure 2 and table $\mathrm{S} 2$. 


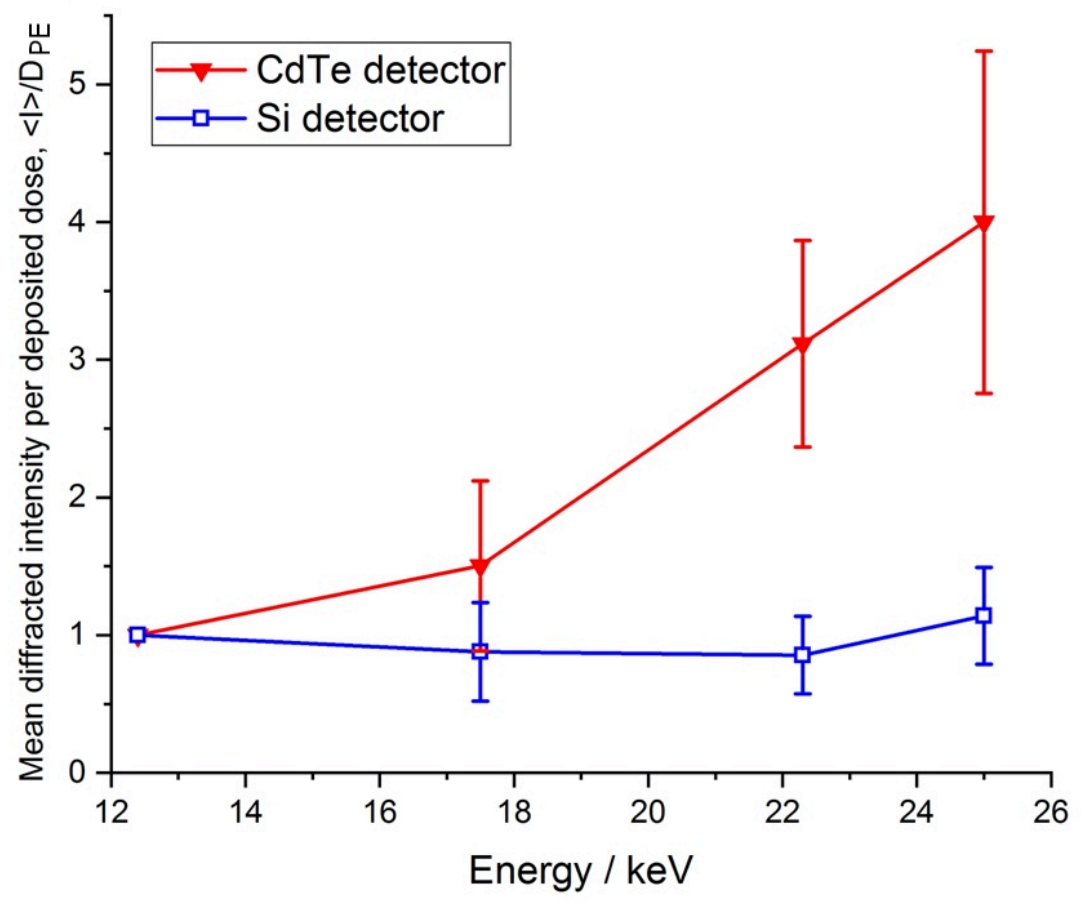

Figure S4: Increase in the diffracted intensity per unit deposited dose, $<\mathrm{I}>/ \mathrm{D}_{\mathrm{PE}}$, as a function of energy. Data shown are averaged from 29 data series (22 recorded using CdTe Eiger and 7 using the Si Pilatus) with the standard deviation at each energy shown as error bars.

\section{References appearing in supplementary material only}

D. Liebschner, P. V. Afonine, M. L. Baker, G. Bunkóczi, V. B. Chen, T. I. Croll, B. Hintze, L.W. Hung, S. Jain, A. J. McCoy, N. W. Moriarty, R. D. Oeffner, B. K. Poon, M. G. Prisant, R. J. Read, J. S. Richardson, D. C. Richardson, M. D. Sammito, O. V. Sobolev, D. H. Stockwell, T. C. Terwilliger, A. G. Urzhumtsev, L. L. Videau, C. J. Williams, and P. D. Adams Acta Cryst. (2019). D75, 861-877 